\title{
On an Alternative View to Complex Calculus
}

\section{Sajedeh Norozpour}

\author{
Submitted to the \\ Institute of Graduate Studies and Research \\ in partial fulfillment of the requirements for the degree of
}

Doctor of Philosophy

in

Mathematics

Eastern Mediterranean University

August 2018

Gazimağusa, North Cyprus 
Approval of the Institute of Graduate Studies and Research

Assoc.Prof. Dr. Ali Hakan Ulusoy

Acting Director

I certify that this thesis satisfies the requirements as a thesis for the degree of Doctor of Philosophy in Mathematics.

Prof. Dr. Nazim Mahmudov

Chair, Department of Mathematics

We certify that we have read this thesis and that in our opinion, it is fully adequate, in scope and quality, as a thesis of the degree of Doctor of Philosophy in Mathematics.

Prof. Dr. Ağamirza Bashirov

Supervisor

Examining Committee

1. Prof.Dr.Ağamirza Bashirov

2. Prof.Dr.Hüseyin Aktuğlu

3. Prof.Dr.Nazim Mahmudov

4. Prof.Dr.Emine Misirlı

5. Prof.Dr.Haluk Hüseyin 


\begin{abstract}
A review of complex calculus in use today, shows that new techniques to solve multivalued nature of logarithmic functions that cause lots of difficulties to work on this subject. One such techniques is re-orientation of complex space to the wider space $\mathbb{B}$ which will be explained completely in the following thesis. The aim of this study is to provide alternative complex calculus on multiplicative and bi-geometric cases. First of all, multiplicative complex calculus was accomplished, then, we found that there are still some drawbacks on this method, so bi-geometric case of complex calculus was established and the results showed that the mentioned drawbacks that are demonstrated in this study, do not appear in bi-geometric case. Further research is recommended to asses the Fourier series, Taylor polynomial and Laurent seried in bi-geometric complex calculus.
\end{abstract}

Keywords: Newtonian Calculus, Bi-geometric complex calculus, Logarithm, Multiplicative complex calculus. 


\section{öz}

Günümüzde kullanımda olan karmaşık kalkülüs (analiz yada hesap) incelemesi, logaritmik fonksiyonların çok değerli doğasını çözecek olan yeni tekniklerin, çok fazla zorluğa neden olduğunu göstermektedir. Bu tür tekniklerden birisi, ilerki kısımlarda detaylı bir şekilde açıklanacak olan, daha geniş bir B’ye karmaşık uzayin yeniden uyarlanmasidir. Bu çalışmadaki amaç, çarpımsal ve bi-geometrik durumlar üzerinde alternatif bir karmaşık analizi sağlamaktır. İlk olarak, çarpımsal karmaşık analiz başarı ile gerçekleştirildi. Ancak daha sonra, bu yöntemde hala bazı sakıncalı durumların bulunduğunu gördük, bu nedenle bi-geometrik karmaşık matematiksel durumu(model) oluşturuldu, ve sonuçlar gösterdiki, Bi-geometrik durumda (modelde) daha önce gözlemlenen sakıncalı durumlar ortadan kalktı. Sonuç olarak gördük ki, bi-geometrik karmaşık hesapta dizilen Fourier serileri, Taylor polinomu ve Laurent'i değerlendirmek için daha fazla araştırma yapılması gerekir.

Anahtar Kelimeler: Newton Analizi, Bi-geometrik karmaşık hesabı, Logaritma, Çarpımsal karmaşık hesap. 


\section{ACKNOWLEDGEMENT}

Firstly, I would like to express my sincere gratitude to my supervisor Prof.Dr.Agamirza Bashirov for the continuous support of my $\mathrm{PhD}$ study and related research, for his patient, motivation and immense knowledge.

Besides my advisor, I would like to thank the rest of my thesis committee for their comments and encouragement.

My sincere thanks also goes to all professors and staffs in the department of Mathematics who provided me an opportunity to join their team, without their support, it would not be possible to conduct this research.

Last but not the least, I would like to thank my family and my beloved one for supporting me spiritually throughout writing this thesis. 


\section{TABLE OF CONTENTS}

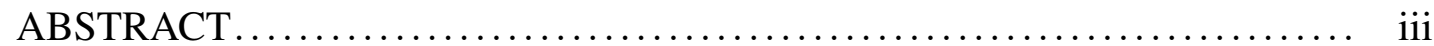

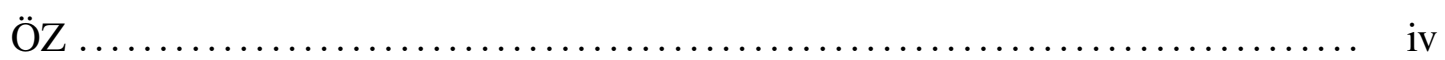

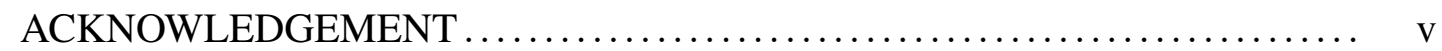

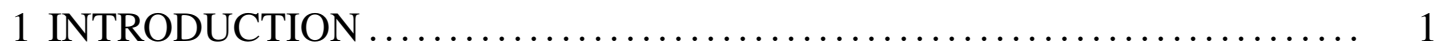

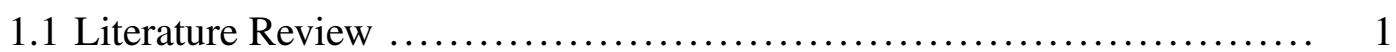

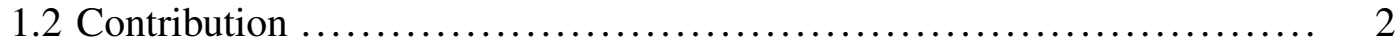

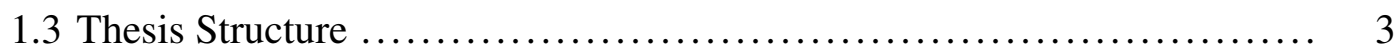

2 MULTIPLICATIVE REAL CALCULUS. ............................. 4

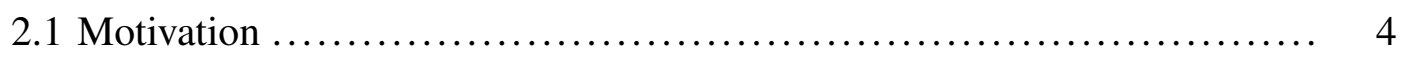

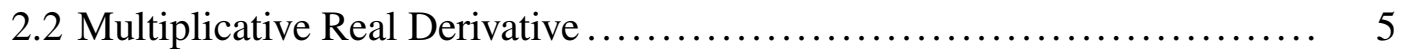

2.3 Multiplicative Real Integral $\ldots \ldots \ldots \ldots \ldots \ldots \ldots \ldots \ldots \ldots \ldots \ldots \ldots \ldots \ldots$

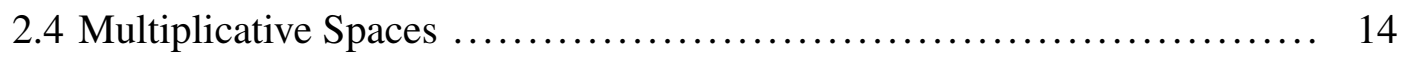

3 MULTIPLICATIVE COMPLEX CALCULUS $\ldots \ldots \ldots \ldots \ldots \ldots \ldots \ldots \ldots$

3.1 Complex Multiplicative Differentiation ........................... 16

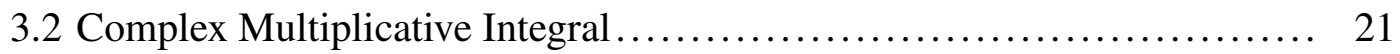

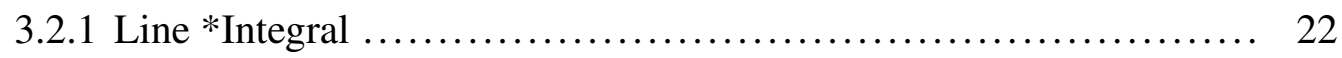

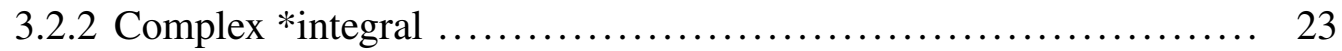

3.2 .3 Properties of Complex *Integral $\ldots \ldots \ldots \ldots \ldots \ldots \ldots \ldots \ldots \ldots \ldots . \ldots \ldots$

4 RIEMANN SURFACE OF COMPLEX LOGARITHM ................. 32

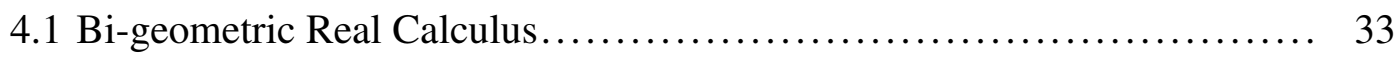

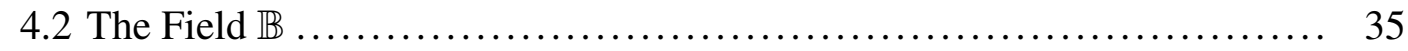

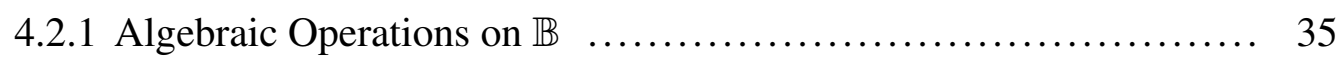




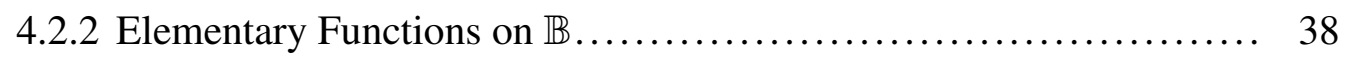

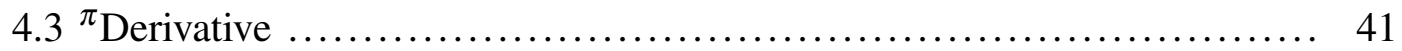

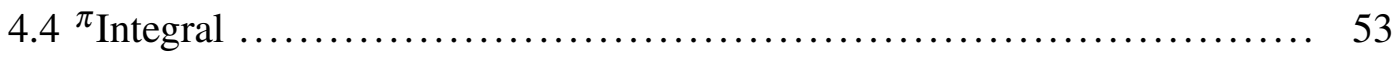

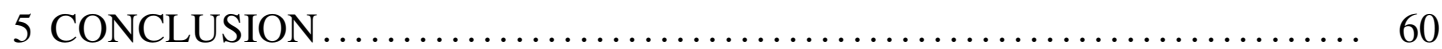

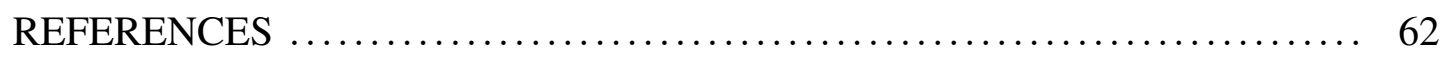




\section{Chapter 1}

\section{INTRODUCTION}

\subsection{Literature Review}

The development of mathematical sciences has been an incredible success through the introduction of non-Newtonian calculi, which are alternatives to the classical calculus of Newton and Leibnitz. A wide variety of mathematical tools are provided by nonNewtonian calculi and researchers in different areas such as sciences, engineering and mathematics are considering these tools. The non-Newtonian calculi were created in the period $1967-1970$ by Michael Grossman and Robert Katz[1]. There are infinite family of calculi which include the classical Newtonian calculus, Geometric calculus, Bi-geometric calculus, harmonic and multiplicative calculi. The non-Newtonian calculi are different from the classical calculus since in them the basic reference function is non-linear, for example, in multiplicative and geometric calculi, it is exponential function and in bi-geometric calculus is power functions. Derivative and integral as two basic operations in analysis and calculus are defined for each calculus. In fact, Michael Grossman and Robert Katz introduced multiplicative calculus by giving definitions of derivative and integral by moving the role of subtraction and addition to division and multiplication, respectively. The concept of multiplicative calculus was well established in [2] which provides interesting aspects of the known phenomena of mathematics and provides achievement in some areas such as numerical analysis [38], fractional [11, 12] and complex analysis [13-17]. Additionally, it was used by lots of researchers in different topics like economics [18-20], image processing[20, 22], functional analysis[23, 24], rates of return, growth processing and signal processing 
[25-28]. The advantages of using multiplicative calculus rather than the classical one in some applications are demonstrated by Nico Persch, Christopher Schroers, Simon Setzer and Joachim Weickert in their two articles [29, 30]. Many applications on non-Newtonian calculus have been done by Agamirza Bashirov, Mustafa Riza, Emine Misirli and Ali Uzer and they showed that some problems from different fields can be modeled more efficiently in the multiplicative case. First of all, multiplicative derivatives and integral were defined in real case which was applicable to strictly positive or negative functions and using multiplicative operations for functions with both positive and negative values faced difficulties. This drawback leads to define an extension of multiplicative operations to complex valued functions and create complex multiplicative calculus [16]. Definitions of complex multiplicative integral and derivative has been written in [13, 14]. It is an interesting fact that, in any non-Newtonian calculus, derivative and integral are expressed in term of classical derivative and classical integral and fundamental theorem which demonstrates the invertibility of them, is established.

\subsection{Contribution}

In this study, we are going to introduce multiplicative integral and multiplicative derivative on complex valued functions. One of the inconveniences of research in the field of complex calculus or complex analysis is the multi-valued nature of some complex functions as logarithmic which is caused by the non unique infinitely countable polar representation of complex numbers. Therefore, distinct polar representation of a complex number create multiple values for these functions, consequently, there be lack of space in the complex plane.

To remove this insufficiency, we define generalized complex space denoted by $\mathbb{B}$ and 
specify different polar representation of complex number over $\mathbb{B}$. In fact, $\mathbb{B}$ is a Riemann surface of complex log-function written in algebraic form.

\subsection{Thesis Structure}

In the present research, chapters are mainly divided into several sections; by way of explanation, introduction, preliminaries and theorems on multiplicative real calculus, multiplicative complex calculus, multiplicative calculus over generalized complex space and special functions on it, and finally conclusion.

The thesis is structured as follows:

Chapter two consists some preliminaries, definitions and theorems related to multiplicative calculus in the real case, multiplicative integral and derivative and their relation with classical derivative and integral and restriction of these definitions to strictly positive or negative functions.

In chapter 3, to eliminate the mentioned restriction, definitions of multiplicative integral and multiplicative derivative are carried out over complex space.

Chapter 4 includes generalized complex space, single valued complex functions and multiplicative and bigeometric calculus over $\mathbb{B}$. Finally, the conclusion of this study is provided. 


\section{Chapter 2}

\section{MULTIPLICATIVE REAL CALCULUS}

\subsection{Motivation}

Imagine your parents created a saving account when you were born. They deposited $\$ a$ with an interest rate of $\% b$ compounded yearly. Clearly, the amount of money after a year is $\$ a+\frac{b}{100} a$, a month is $\$ a+\frac{b}{12(100)} a$ and after a day is $\$ a+\frac{b}{365(100)} a$, by this assumption that a year is 12 months and 365 days. Let $\frac{b}{100} a=c_{1}, \frac{b}{12(100)} a=c_{2}$ and $\frac{b}{365(100)} a=c_{3}$ then the average growth factor is $\frac{a+c_{1}}{a}$ yearly, $\frac{a+c_{2}}{a}$ monthly and $\frac{a+c_{3}}{a}$ daily. Now, let $\rho$ be annual growth factor then

$$
\rho=\frac{a+c_{1}}{a}=\left(\frac{a+c_{2}}{a}\right)^{\frac{1}{12}}=\left(\frac{a+c_{3}}{a}\right)^{\frac{1}{365}} .
$$

Using information above, if we assume that $f$ is a function which describes the amount of deposited money at the time $x$ then to find growth factor we need to evaluate

$$
\lim _{\Delta x \rightarrow 0}\left(\frac{f(x+\Delta x)}{f(x)}\right)^{\frac{1}{\Delta x}}
$$

which shows that how many times $f(x)$ changes at the time $x$. If we compare limit (2.1) with the classical derivative formula below

$$
\lim _{\Delta x \rightarrow 0} \frac{f(x+\Delta x)-f(x)}{\Delta x} .
$$

It can be easily found that the role of subtraction and division in (2.2) is derived to division and raising to power respectively, in (2.1).

The limit (2.1) is called multiplicative derivative, or briefly *derivative, which will be 
discussed in the next section.

\subsection{Multiplicative Real Derivative}

Before starting this section, let us mention that multiplicative derivative is suitable for positive or negative functions. So, multiplicative real derivative is defined just for positive functions and negative functions will be studied in the complex case.

Definition 1. Let $f: \mathbb{R} \rightarrow \mathbb{R}$ be positive or negative function, then *derivative of $f$ is defined as follows:

$$
f^{*}(x)=\lim _{\Delta x \rightarrow 0}\left(\frac{f(x+\Delta x)}{f(x)}\right)^{\frac{1}{\Delta x}}
$$

Remark 1. $f^{*}(x)$ can be written in terms of Newtonian derivative as

$$
f^{*}(x)=e^{(\ln f(x))^{\prime}} .
$$

Proof: Using definition of multiplicative derivative, it can be easily seen that,

$$
\begin{aligned}
f^{*}(x) & =\lim _{\Delta x \rightarrow 0}\left(\frac{f(x+\Delta x)}{f(x)}\right)^{\frac{1}{\Delta x}} \\
& =\lim _{\Delta x \rightarrow 0}\left(1+\frac{f(x+\Delta x)-f(x)}{f(x)}\right)^{\frac{f(x)}{f(x+\Delta x)-f(x)} \cdot \frac{f(x+\Delta x)-f(x)}{\Delta x} \cdot \frac{1}{f(x)}} \\
& =e^{\frac{f^{\prime}(x)}{f(x)}}
\end{aligned}
$$

Higher *derivative of $f$ can be expressed as

$$
f^{*(n)}=e^{(\ln f(x))^{(n)}(x)}, \quad n=2,3,4, \ldots
$$

So, due to these definitions, a function $f: A \subset \mathbb{R} \rightarrow \mathbb{R}$ is said to be $*$ differentiable at $x$ is $f$ is positive and differentiable at $x$.

Definition 2. A function $f: A \subset \mathbb{R} \rightarrow \mathbb{R}$ is said to be *differentiable on $A$ if it is *differentiable at any point of $A$. 
Definition 3. Recall that, the *partial derivatives of a function $f$ with two variables $x$ and $y$ are denoted by $f_{x}^{*}$ and $f_{y}^{*}$ by considering $y$ and $x$ as constant, respectively.

Lemma 1. *derivative has the following properties:

(i) $(c f)^{*}(x)=f^{*}(x)$,

(ii) $(f g)^{*}(x)=f^{*}(x) g^{*}(x)$,

(iii) $(f / g)^{*}(x)=f^{*}(x) / g^{*}(x)$,

(iv) $\left(f^{h}\right)^{*}(x)=f^{*}(x)^{h(x)} \cdot f(x)^{h^{\prime}(x)}$,

(v) $(f \circ h)^{*}(x)=f^{*}(h(x))^{h^{\prime}(x)}$,

where $c$ is a positive constant, $f$ and $g$ both are $*$ differentiable and $h$ is differentiable.

Proof: (i) Due to the definition of *derivative,

$$
\begin{aligned}
(c f)^{*}(x) & =e^{(\ln (c f))^{\prime}(x)} \\
& =e^{(\ln c+\ln f)^{\prime}(x)} \\
& =e^{(\ln f)^{\prime}(x)} \\
& =f^{*}(x) .
\end{aligned}
$$

(ii) We have,

$$
\begin{aligned}
(f g)^{*}(x) & =e^{(\ln (f g))^{\prime}(x)} \\
& =e^{(\ln f)^{\prime}(x)+(\ln g)^{\prime}(x)} \\
& =e^{(\ln f)^{\prime}(x)} e^{(\ln g)^{\prime}(x)} \\
& =f^{*}(x) g^{*}(x) .
\end{aligned}
$$


(iii) It can be derived by,

$$
\begin{aligned}
(f / g)^{*}(x) & =e^{(\ln (f / g))^{\prime}(x)} \\
& =e^{(\ln f)^{\prime}(x)-(\ln g)^{\prime}(x)} \\
& =e^{(\ln f)^{\prime}(x)} / e^{(\ln g)^{\prime}(x)} \\
& =f^{*}(x) / g^{*}(x) .
\end{aligned}
$$

(iv) Part (iv) can be proved as below;

$$
\begin{aligned}
\left(f^{h}\right)^{*}(x) & =e^{\left(\ln f^{h}\right)^{\prime}(x)} \\
& =e^{(h \ln f)^{\prime}(x)} \\
& =e^{h(x)(\ln f)(x)+h(x)(\ln f)^{\prime}(x)} \\
& =e^{(\ln f)^{h^{\prime}(x)}} e^{(\ln f)^{\prime}(x) h(x)} \\
& =f^{*}(x)^{h(x)} \cdot f(x)^{h^{\prime}(x)}
\end{aligned}
$$

(v) The *derivative of composition of two functions can be found as follows;

$$
\begin{aligned}
(f \circ h)^{*}(x) & =e^{(\ln f \circ h)^{\prime}(x)} \\
& =e^{\frac{f^{\prime}(h(x)) h^{\prime}(x)}{f(h(x))}} \\
& =e^{h^{\prime}(x)(\ln f)^{\prime}(h(x))} \\
& =f^{*}(h(x))^{h^{\prime}(x)}
\end{aligned}
$$

Example 1. Let $f(x)=c$ be a constant function. Then the *derivative of $f$ is as follows:

$$
\begin{aligned}
f^{*}(x) & =e^{(\ln c)^{\prime}(x)} \\
& =e^{0} \\
& =1 .
\end{aligned}
$$


It can be deduced that, the role of zero in Newtonian calculus is derived to $1 \mathrm{in} *$ calculus.

Example 2. Consider $f(x)=e^{x} \ln x$, then the *derivative of $f$, by using Lemma 1.(iii) is :

$$
\begin{aligned}
f^{*}(x) & =e^{\left(\ln e^{x}\right)^{\prime}} e^{(\ln (\ln x))^{\prime}} \\
& =e^{1} e^{\frac{1}{x \ln x}} \\
& =e^{1+\frac{1}{x \ln x}}
\end{aligned}
$$

Next in time, we establish some sequels of Newtonian calculus in term of *derivative.

Theorem 1. (Multiplicative Mean Value Theroem)[2] Assume that function $f$ is continuous on $[a, b]$ and $*$ differentiable on $(a, b)$, then there exists $a<c<b$ such that

$$
\frac{f(b)}{f(a)}=f^{*}(c)^{b-a}
$$

Corollary 1. (Multiplicative tests for Monotonicity)[2] Assume that function $f$ is *differentiable on $(a, b)$, then the followings hold.

(a) if $f^{*}(x)>1, \forall x \in(a, b)$, then $f$ increases strictly.

(b) if $f^{*}(x)<1, \forall x \in(a, b)$, then $f$ decreases strictly.

(c) if $f^{*}(x) \geq 1, \forall x \in(a, b)$, then $f$ is increasing function.

(d) if $f^{*}(x) \leq 1, \forall x \in(a, b)$, then $f$ is decreasing function.

Corollary 2. (Multiplicative tests for Local Extremum) Assume that function $f$ is twice $*$ differentiable on $(a, b)$. 
(a) If $c \in(a, b)$ is a local extremum of $f$, then $f^{*}(c)=1$.

(b) If $f^{*}(c)=1$ and $f^{* *}(c)>1$, then $f$ takes its local minimum at $c$.

(c) If $f^{*}(c)=1$ and $f^{* *}(c)<1$, then $f$ takes its local maximum at $c$.

Note. The facts above can be easily found from Newtonian Calculus by deriving subtraction to division and division to the raising to a power.

Theorem 2. (Multiplicative Chain Rule) Let $f(y(x), z(x))$ be a function with continuous partial *derivatives. If $y$ and $z$ are differentiable on $(a, b)$ such that $f(y(x), z(x))$ is defined for every $x \in(a, b)$, then

$$
\frac{d^{*} f(y(x), z(x))}{d x}=f_{y}^{*}(y(x), z(x))^{y^{\prime}(x)} f_{z}^{*}(y(x), z(x))^{z^{\prime}(x)} .
$$

\section{Proof:}

$$
\begin{aligned}
\frac{d^{*} f(y(x), z(x))}{d x} & =e^{\frac{d(\ln f(y(x), z(x)))}{d x}} \\
& =e^{\frac{d(\ln f(y(x), z(x)))}{d y} \cdot \frac{d y}{d x}+\frac{d(\ln f(y(x), z(x)))}{d z} \cdot \frac{d z}{d x}} \\
& =e^{\frac{(d(\ln f(y(x), z(x)))}{d y} \cdot \frac{d y}{d x}} e^{\frac{d(\ln f(y(x), z(x)))}{d z} \cdot \frac{d z}{d x}} \\
& =e^{(\ln f(y(x), z(x)))_{y}^{\prime} y^{\prime}(x)} e^{(\ln f(y(x), z(x)))_{z}^{\prime} z^{\prime}(x)} \\
& =f_{y}^{*}(y(x), z(x))^{y^{\prime}(x)} f_{z}^{*}(y(x), z(x))^{z^{\prime}(x)} .
\end{aligned}
$$




\subsection{Multiplicative Real Integral}

Let $\rho=\left\{x_{1}, x_{2}, \ldots, x_{n}\right\}$ be a partition on $[a, b]$ and consider *product below,

$$
P(f, \rho)=\prod_{i=1}^{n}\left|f\left(c_{i}\right)\right|^{\left(x_{i}-x_{i-1}\right)},
$$

where $f$ is positive bounded function on $[a, b]$. if (2.3) converges when the mesh $\rho$ goes to zero, then the function $f$ is said to be *integrable and the limit of *product (2.3) is denoted by $\int_{a}^{b} f(x)^{d x}$.

Theorem 3. Let $f$ be positive and Riemann integrable on $[a, b]$. then $f$ is *integrable on $[a, b]$ and

$$
\int_{a}^{b} f(x)^{d x}=e^{\int_{a}^{b} \ln f(x) d x}
$$

Proof: This follows from;

$$
\begin{aligned}
\int_{a}^{b} f(x)^{d x} & =\lim _{n \rightarrow \infty} \prod_{i=1}^{n}\left|f\left(c_{i}\right)\right|^{\left(x_{i}-x_{i-1}\right)} \\
& =\lim _{n \rightarrow \infty} e^{\ln \left(\prod_{i=1}^{n}\left|f\left(c_{i}\right)\right|^{\left(x_{i}-x_{i-1}\right)}\right)} \\
& =\lim _{n \rightarrow \infty} e^{\sum_{i=1}^{n}\left|\ln f\left(c_{i}\right)\right|^{\left(x_{i}-x_{i-1}\right)}} \\
& =e^{\int_{a}^{b} \ln f(x) d x}
\end{aligned}
$$

Lemma 2. The followings are the properties of multiplicative integral.

(a) $\int_{a}^{b}\left(f(x)^{p}\right)^{d x}=\left(\int_{a}^{b} f(x)^{d x}\right)^{p}, p \in \mathbb{R}$

(b) $\int_{a}^{b}(f(x) g(x))^{d x}=\int_{a}^{b} f(x)^{d x} \int_{a}^{b} g(x)^{d x}$

(c) $\int_{a}^{b}(f(x) / g(x))^{d x}=\frac{\int_{a}^{b} f(x)^{d x}}{\int_{a}^{b} g(x)^{d x}}$

(d) $\int_{a}^{b} f(x)^{d x}=\int_{a}^{c} f(x)^{d x} \int_{c}^{b} f(x)^{d x},(a \leq c \leq b)$ 
Proof: (a) using definition we have;

$$
\begin{aligned}
\int_{a}^{b}\left(f(x)^{p}\right)^{d x} & =e^{\int_{a}^{b}\left(\ln f(x)^{p}\right) d x} \\
& =e^{p \int_{a}^{b} \ln f(x) d x} \\
& =e^{\left(\int_{a}^{b} \ln f(x) d x\right)^{p}} \\
& =\left(\int_{a}^{b} f(x)^{d x}\right)^{p}
\end{aligned}
$$

(b) We have;

$$
\begin{aligned}
\int_{a}^{b}(f(x) g(x))^{d x} & =e^{\int_{a}^{b} \ln (f(x) g(x)) d x} \\
& =e^{\int_{a}^{b}(\ln f(x)+\ln g(x)) d x} \\
& =e^{\int_{a}^{b} \ln f(x) d x+\int_{a}^{b} \ln g(x) d x} \\
& =\int_{a}^{b} f(x)^{d x} \int_{a}^{b} g(x)^{d x}
\end{aligned}
$$

(c) It can be easily shown that;

$$
\begin{aligned}
\int_{a}^{b}(f(x) / g(x))^{d x} & =e^{\int_{a}^{b} \ln (f(x) / g(x)) d x} \\
& =e^{\int_{a}^{b}(\ln f(x)-\ln g(x)) d x} \\
& =e^{\int_{a}^{b} \ln f(x) d x-\int_{a}^{b} \ln g(x) d x} \\
& =\frac{e^{\int_{a}^{b} \ln f(x) d x}}{e^{\int_{a}^{b} \ln g(x) d x}} \\
& =\frac{\int_{a}^{b} f(x)^{d x}}{\int_{a}^{b} g(x)^{d x}}
\end{aligned}
$$


(d) Clearly,

$$
\begin{aligned}
\int_{a}^{b} f(x)^{d x} & =e^{\int_{a}^{b} \ln f(x) d x} \\
& =e^{\int_{a}^{c} \ln f(x) d x+\int_{c}^{b} \ln f(x) d x} \\
& =e^{\int_{a}^{c} \ln f(x) d x} e^{\int_{c}^{b} \ln f(x) d x} \\
& =\int_{a}^{c} f(x)^{d x} \int_{c}^{b} f(x)^{d x}
\end{aligned}
$$

Theorem 4. (Fundamental Theorem of Multiplicative Calculus)

a) Assume $f:[a, b] \rightarrow \mathbb{R}$ is *differentiable and $f^{*}$ is *integrable then;

$$
\int_{a}^{b} f^{*}(x)^{d x}=\frac{f(b)}{f(a)} .
$$

b) Assume $f:[a, b] \rightarrow \mathbb{R}$ is *integrable and $F(x)=\int_{a}^{x} f(t)^{d t},\left(a \leq x^{\natural} b\right)$. If $\mathrm{f}$ is continuous at $\xi \in[a, b]$, then $F$ is $*$ differentiable at $\xi$ and $F^{*}(\xi)=f(\xi)$.

\section{Proof: a)}

$$
\begin{aligned}
\int_{a}^{b} f^{*}(x)^{d x} & =\int_{a}^{b}\left(e^{(\ln f)^{\prime}}\right)^{d x} \\
& =e^{\int_{a}^{b}(\ln f)^{\prime}(x) d x} \\
& =e^{\ln f(b)-\ln f(a)} \\
& =e^{\ln \frac{f(b)}{f(a)}} \\
& =\frac{f(b)}{f(a)}
\end{aligned}
$$

b) As it is given, $F(x)=\int_{a}^{x} f(t)^{d t}=e^{\int_{a}^{x} \ln f(t) d t}$. Since $f$ is continuous so is $F$ and 


$$
\begin{aligned}
F^{*}(\xi) & =e^{(\ln F)^{\prime}(\xi)} \\
& =e^{\frac{F^{\prime}(\xi)}{F(\xi)}} \\
& =e^{\frac{e^{\int_{a}^{\xi} \ln f(t) d t} \cdot \ln f(\xi)}{e^{\int_{a}^{\xi} \ln f(t) d t}}} \\
& =e^{\ln f(\xi)} \\
& =f(\xi) .
\end{aligned}
$$

Theorem 5. (Multiplicative Integration by Parts) Assume $f:[a, b] \rightarrow \mathbb{R}$ is *differentiable and $g:[a, b] \rightarrow \mathbb{R}$ is differentiable, then $f^{g}$ is *integrable on $[a, b]$ and,

$$
\int_{a}^{b}\left(f^{*}(x)^{g(x)}\right)^{d x}=\frac{f(b)^{g(b)}}{f(a)^{g(a)}} \frac{1}{\int_{a}^{b}\left(f(x)^{g^{\prime}(x)}\right)^{d x}}
$$

Proof: We know that

$$
\left(f^{g}\right)^{*}(x)=f^{*}(x)^{h(x)} \cdot f(x)^{h^{\prime}(x)} .
$$

Integrate both sides, we will get

$$
\begin{aligned}
\int_{a}^{b}\left(f^{g}\right)^{*}(x)^{d x} & =\int_{a}^{b}\left(f^{*}(x)^{h(x)} \cdot f(x)^{h^{\prime}(x)}\right)^{d x} \\
& =\int_{a}^{b}\left(f^{*}(x)^{h(x)}\right)^{d x} \int_{a}^{b}\left(f(x)^{h^{\prime}(x)}\right)^{d x}
\end{aligned}
$$

By using Fundamental theorem of multiplicative calculus we can find that,

$$
\frac{f(b)^{g(b)}}{f(a)^{g(a)}}=\int_{a}^{b}\left(f^{*}(x)^{h(x)}\right)^{d x} \int_{a}^{b}\left(f(x)^{h^{\prime}(x)}\right)^{d x}
$$

which gives us the result. 
Lemma 3. The following integrals hold.

a) $\int_{a}^{a} f(x)^{d x}=1$

b) $\int_{a}^{b} f(x)^{d x}=\left(\int_{b}^{a} f(x)^{d x}\right)^{-1}$.

\section{Proof:}

a) $\int_{a}^{a} f(x)^{d x}=e^{\int_{a}^{a} \ln f(x) d x}=e^{0}=1$.

b) It can be obtained as follows ;

$$
\begin{aligned}
\int_{a}^{b} f(x)^{d x} & =e^{\int_{a}^{b} \ln f(x) d x} \\
& =e^{-\int_{b}^{a} \ln f(x) d x} \\
& =\left(e^{\int_{b}^{a} \ln f(x) d x}\right)^{-1} \\
& =\left(\int_{b}^{a} f(x)^{d x}\right)^{-1}
\end{aligned}
$$

\subsection{Multiplicative Spaces}

Definition 4. Given $x \in \mathbb{R}^{+}$. The multiplicative absolute value of $x$ is defined as:

$$
|x|^{*}= \begin{cases}x, & \text { if } x \geq 1 \\ x^{-1}, & \text { if } x<1\end{cases}
$$

Remark 2. Properties of *absolute value are as follows:

(a) $|x|^{*} \geq 1$

(b) $\frac{1}{|x|^{*}} \leq x \leq|x|^{*}$

(c) $|x|^{*}=\left|x^{-1}\right|^{*}$

(d) $|x|^{*} \leq y \Longleftrightarrow y^{-1} \leq x \leq y$

(e) $|x y|^{*} \leq|x|^{*}|y|^{*}$ 
Definition 5. (Multiplicative Metric Space) Let $X \neq \emptyset$. A function $d^{*}: X \times X \rightarrow \mathbb{R}^{+}$ is called multiplicative metric on $X$ if it satisfies the followings:

a) $d^{*}(x, y) \geq 1$ and $d^{*}(x, y)=1 \Longleftrightarrow x=y$.

b) $d^{*}(x, y)=d^{*}(y, x)$

c) $d^{*}(x, y) \leq d^{*}(x, z) d^{*}(z, y)$ (multiplicative triangle inequality)

Then the pair $\left(X, d^{*}\right)$ is named multiplicative metric space.

Example 1. Let $X=C^{*}[a, b]$ be the collection of all multiplicative continuous functions on $[a, b]$, let us define metric below

$$
d^{*}(f, g)=\sup _{x \in[a, b]}\left|\frac{f(x)}{g(x)}\right|, \forall f, g \in X
$$

Then, $(X, d)$ is a multiplicative metric space

Definition 6. (Lipcshitz $*$ Condition) Let $\left(X, d^{*}\right)$ be a multiplicative metric space and $f: X \rightarrow X$. one says $f$ satisfies *Lipshitz condition if $\exists \ell>1$ such that $d^{*}(f(x), f(y)) \leq$ $\ell^{d^{*}(x, y)}$ and if $0 \leq \ell<1$ then $f$ is said to be contraction mapping. 


\section{Chapter 3}

\section{MULTIPLICATIVE COMPLEX CALCULUS}

In the chapter ahead, we are going to develop the concepts of $*$ derivative and *integral to complex valued functions. As it is stated, the *derivative of real valued function $f$ is $f^{*}(t)=e^{(\ln o f)^{\prime}(t)}$, if $f$ is strictly positive. The definition still holds if $f$ be strictly negative function and it is in the form of $f^{*}(t)=e^{(\ln |f|)^{\prime}(t)}$. obviously, $f^{*}(t)>0$ for any non vanishing function $f$ and $*$ derivative and $*$ integral of functions with positive and negative values is not applicable in real case since $\mathbb{R}-\{0\}$ should be considered in this case and we know that a continuous function with the range in $\mathbb{R}-\{0\}$ is always positive or negative. This drawback leads to define *derivative and *integral in complex space and construct multiplicative complex calculus. Since passing continuously from positive part to negative part or vice versa is allowed in complex plane.

\subsection{Complex Multiplicative Differentiation}

Noting that, $\log |f|^{\prime}(t)=\ln |f|^{\prime}(t)+i \Theta^{\prime}(t)$ and branch of $f$ may not exists or if exists, it may not be as composition of a branch of $\log$ and $f$. Due to this fact, the *derivative is defined in the base of local behavior of function $f$. In fact, we select a sufficiently small neighborhood $\mathrm{U} \subseteq(a, b)$ of $t$ for which, the branch of $f$ exists on it and it is a composition of branch of $\log$ and $f$. Additionally, by using localization we may use $(\log f)^{\prime}=\frac{f^{\prime}}{f}$ independet on selection of branch of $\log$.

Definition 7. Assume $f$ is nowhere vanishing complex valued function on open $D \subseteq \mathbb{C}$ and let $\mathrm{U} \subseteq D$ is a neighborhood such that the branch of $\log f$ in the form of composition of respective branches of $\log$ and $f$ exist, then the *derivative of $f$ is

$$
f^{*}(z)=e^{\frac{f^{\prime}(z)}{f(z)}}, \forall z \in D .
$$


Consequently, the higher order multiplicative derivatives are defined as:

$$
\left(f^{*}\right)^{(n)}(z)=e^{\left(\frac{f^{\prime}(z)}{f(z)}\right)^{(n-1)}} .
$$

Lemma 4. (Cauchy-Riemann *conditions) Let $z=x+i y=r e^{i \theta}$ and $f(z)=u(z)+$ $i v(z)=R(z) e^{\Theta(z)+2 n \pi}$, where $\Theta$ is any branch of $\arg f$ and $R(z)=\sqrt{u^{2}(z)+v^{2}(z)}$ then,

(a) $R_{x}^{*}(z)=\left(e^{\Theta}\right)_{y}^{*}(z)$

(b) $R_{y}^{*}(z)=\left(e^{-\Theta}\right)_{x}^{*}(z)$

\section{Proof:}

a) To prove $R_{x}^{*}(z)=\left(e^{\Theta}\right)_{y}^{*}(z)$, it is enough to show that $(\ln R)_{x}^{\prime}(z)=\Theta_{y}^{\prime}(z)$, since $R_{x}^{*}(z)=e^{(\operatorname{lnR})_{x}^{\prime}(z)}$ and $\left(e^{\Theta}\right)_{y}^{*}(z)=e^{\left(\ln e^{\Theta}\right)_{y}^{\prime}(z)}$. we divide the proof into two cases :

Case 1: Let $u(z) \neq 0$, then $\ln \mathrm{R}=\ln \sqrt{u^{2}+v^{2}}=\frac{1}{2} \ln \left(u^{2}+v^{2}\right)$ and

$$
(\operatorname{lnR})_{x}^{\prime}=\frac{u u_{x}^{\prime}+v v_{x}^{\prime}}{u^{2}+v^{2}}
$$

From the Cauchy-Riemann conditions we have $u_{x}^{\prime}=v_{y}^{\prime}$ and $u_{y}^{\prime}=-v_{x}^{\prime}$, so ,

$$
\begin{aligned}
(\operatorname{lnR})_{x}^{\prime} & =\frac{u v_{y}^{\prime}-v u_{y}^{\prime}}{u^{2}+v^{2}} \\
& =\frac{1}{1+\frac{v^{2}}{u^{2}}} \frac{u v_{y}^{\prime}-v u_{y}^{\prime}}{u^{2}} \\
& =\left(\tan ^{-1} \frac{v}{u}+\pi k+2 n \pi\right)_{y}^{\prime} \\
& =\Theta_{y}^{\prime}(z),
\end{aligned}
$$

where $k \in\{-1,0,1\}$. 
Case 2: Let $u(z)=0$, then $v(z) \neq 0$ and

$$
\begin{aligned}
(\operatorname{lnR})_{x}^{\prime} & =\frac{v_{x}^{\prime}(z)}{v(z)} \\
& =\frac{-u_{y}^{\prime}(z)}{v(z)} \\
& =\Theta_{y}^{\prime}(z) .
\end{aligned}
$$

b) it is sufficient to prove that $(\operatorname{lnR})_{y}^{\prime}(z)=-\Theta_{x}^{\prime}(z)$ and the rest is as the same as part (a) .

Lemma 5. Cauchy-Riemann * conditions in polar coordinate are in the form below :

(a) $R_{\theta}^{*}(z)=\left(e^{-\Theta}\right)_{r}^{*}(z)^{r}$

(b) $R_{r}^{*}(z)^{r}=\left(e^{\Theta}\right)_{\theta}^{*}(z)$

Proof: Since $R_{\theta}^{*}(z)=e^{(\operatorname{lnR})_{\theta}^{\prime}(z)},\left(e^{-\Theta}\right)_{r}^{*}(z)^{r}=e^{(-\Theta)_{r}^{\prime} r},\left(e^{\Theta}\right)_{\theta}^{*}(z)=e^{(\Theta)_{r}^{\prime}(z)}$ and $R_{r}^{*}(z)^{r}=$ $e^{(\operatorname{lnR})_{r}^{\prime} r}$, it suffices to prove $(\operatorname{lnR})_{\theta}^{\prime}(z)=-r(\Theta)_{r}^{\prime}$ and $\Theta_{\theta}^{\prime}=r(\operatorname{lnR})_{r}^{\prime}$. Now, to do so let $x=r \cos \theta$ and $y=r \sin \theta$ then,

a)

$$
\begin{aligned}
(\operatorname{lnR})_{\theta}^{\prime}(z) & =(\operatorname{lnR})_{x}^{\prime} x_{\theta}^{\prime}+(\operatorname{lnR})_{y}^{\prime} y_{\theta}^{\prime} \\
& =-\Theta_{y}^{\prime}(z) \cdot r \sin \theta-\Theta_{x}^{\prime}(z) r \cos \theta \\
& =-r\left(\Theta_{y}^{\prime} y_{r}^{\prime}+\Theta_{x}^{\prime} x_{r}^{\prime}\right) \\
& =-r(\Theta)_{r}^{\prime} .
\end{aligned}
$$


b)

$$
\begin{aligned}
\Theta_{\theta}^{\prime} & =\Theta_{x}^{\prime} x_{\theta}^{\prime}+\Theta_{y}^{\prime} y_{\theta}^{\prime} \\
& =-(\operatorname{lnR})_{y}^{\prime}(-r \sin \theta)+(\ln )_{x}^{\prime}(r \cos \theta) \\
& =r\left((\operatorname{lnR})_{y}^{\prime} y_{r}^{\prime}+(\ln \mathrm{R})_{x}^{\prime} x_{r}^{\prime}\right) \\
& =r(\operatorname{lnR})_{r}^{\prime} .
\end{aligned}
$$

Lemma 6. Some of properties of *derivative in complex case are as follows:

(a) $[c f]^{*}(z)=f^{*}(z) \quad c \in \mathbb{C}$

(b) $[f g]^{*}(z)=f^{*}(z) g^{*}(z)$

(c) $[f / g]^{*}(z)=f^{*}(z) / g^{*}(z)$.

Although these properties are extension of properties in real case to complex case, it is not possible to extend all properties of real *derivative to complex form like

$$
\left[f^{g}\right]^{*}(x)=\left(f^{*}(x)\right)^{g(x)}(f(x))^{g^{\prime}(x)}
$$

and

$$
[f \circ g]^{*}(x)=\left(f^{*}(g(x))\right)^{g^{\prime}(x)}
$$

since they include multi-valued functions. These equalities can be stated as :

(d) $\left[e^{g \log f}\right]^{*}(z) \subseteq e^{g(z) \log f^{*}(z)} e^{g^{\prime}(z) \log f(z)}$ where each branch of $\left[e^{g \log f}\right]^{*}(z)$ can be expressed as production of some branch values of $e^{g(z) \log f^{*}(z)}$ and $e^{g^{\prime}(z) \log f(z)}$.

(e) $[f \circ g]^{*}(z) \in e^{g^{\prime}(z) \log f^{*}(g(z))}$ where $[f \circ g]^{*}(z)$ is equal to some branch values of $e^{g^{\prime}(z) \log f^{*}(g(z))}$. 
Proof: The proof of parts $a, b$ and $c$ are as the same as properties of *derivative in real case. Now, we will prove parts $d$ and $e$.

(d) we have

$$
\begin{aligned}
{\left[e^{g \log f}\right]^{*}(z) } & =e^{g^{\prime}(z) \log f(z)} e^{g(z) \log f^{*}(z)} . \\
& =e^{g^{\prime}(z) \log f(z)} e^{g(z) \log e^{f^{\prime}(z) / f(z)}} \\
& =e^{g^{\prime}(z) \log f(z)} e^{g(z) f^{\prime}(z) / f(z)} .
\end{aligned}
$$

Let $w=f^{\prime}(z) / f(z)$. Generally, we have $\log e^{w} \neq w$ and the equality $\mathscr{L} e^{w}=w$ holds if $\mathscr{L}$ be a branch of $\log$ where the imaginary part of $w$ falls into $(\alpha, \alpha+2 \pi]$. Using this fact, $\left[e^{g \log f}\right]^{*}(z) \subseteq e^{g(z) \log f^{*}(z)} e^{g^{\prime}(z) \log f(z)}$ and it is going to be equality if the range of imaginary part of $f^{\prime}(z) / f(z)$ be $(\alpha, \alpha+2 \pi]$.

(e)

$$
\begin{aligned}
{[f \circ g]^{*}(z) } & =f^{*}(g(z))^{g^{\prime}(z)} \\
& =e^{g^{\prime}(z) \log f^{*}(g(z))} .
\end{aligned}
$$

Again, using the fact mentioned in part $(d)$ if $w=f^{\prime}(g(z)) / f(g(z))$ and range of imaginary part of $w$ falls into $(\alpha, \alpha+2 \pi]$ then the equality above holds, otherwise we have

$$
[f \circ g]^{*}(z) \subset e^{g^{\prime}(z) \log f^{*}(g(z))} .
$$

Example 1. Let $f(z)=e^{c z}, z \in \mathbb{C}$ and $c=$ const $\in \mathbb{C}$ then,

$$
f^{*}(z)=e^{f^{\prime}(z) / f(z)}=e^{c e^{c z} / e^{c z}}=e^{c}, z \in \mathbb{C},
$$

It implies that $f(z)=e^{c z}$ plays role of $g(z)=a z$ in Newtonian calculus where $a=e^{c}$. Note: Clearly, the function in example (1) is entire function and as it can be seen the *derivative of $f$, which is $e^{c}$ is again entire, but it does not mean that the *derivative of any entire function is entire. To show that, consider example below. 
Example 2. Assume $f(z)=z, z \in \mathbb{C}$, (which is entire) then,

$$
f^{*}(z)=e^{f^{\prime}(z) / f(z)}=e^{1 / z}, z \in \mathbb{C} \backslash\{0\},
$$

which shows that the $*$ derivative of entire function $f$ has singularity at $z=0$, implying that it is not entire. In the following up, we will show that the *derivative of multivalued functions may be multi-valued or single-valued.

Example 3. Let

$$
f(z)=\log z, z \in \mathbb{C} \backslash\{0\} .
$$

We know that $f(z)$ is multi-valued function with a branch point at $z=0$. Its *derivative is

$$
f^{*}(z)=e^{1 /(z \log z)}
$$

which is still multi-valued with a branch point at $z=0$.

Example 4. Let

$$
f(z)=e^{z \log z}, z \in \mathbb{C} \backslash\{0\}
$$

which is multi-valued function with a branch point at $z=0$, but its *derivative

$$
f^{*}(z)=e^{f^{\prime}(z) / f(z)}=e^{1+\log z}=e z
$$

is single-valued with removable singularity at $z=0$.

\subsection{Complex Multiplicative Integral}

In the section forward, we want to develop multiplicative integral to complex case. To do so, we need to establish *line integral and fundamental theorem of calculus. 


\subsubsection{Line *Integral}

Let $f$ be a positive function with two variables $x$ and $y$ on open connected set $D \subset$ $\mathbb{R} \times \mathbb{R}$ and let $C$ be a piecewise smooth simple curve in the domain on $f$. Let $\mathscr{P}=$ $\left\{P_{0}, \ldots, P_{m}\right\}$ be a partition on $C$ and $\left(\zeta_{k}, \eta_{k}\right)=\xi_{k}$ where $p_{m}=\left(x_{m}, y_{m}\right)$. Define the integral product as $P(f, \mathscr{P})=\prod_{k=1}^{m} f\left(\xi_{k}\right)^{\Delta s_{k}}$ where $\Delta s_{k}$ is arclength of $C$ from $P_{k-1}$ to $P_{k}$. Then, $\lim _{\max \left(\Delta s_{1} \ldots, \Delta s_{m}\right) \rightarrow 0} P(f, \mathscr{P})$ is called *line integral of $f$ along $C$ and it is denoted by $\int_{C} f(x, y)^{d s}$ and it is defined as $\int_{C} f(x, y)^{d s}=e^{\int_{C} \ln f(x, y) d s}$. The *line integrals along $x$ - axis and $y$-axis can be defined in the similar way as follows;

$$
\begin{aligned}
& \int_{C} f(x, y)^{d x}=e^{\int_{C} \ln f(x, y) d x} \\
& \int_{C} f(x, y)^{d y}=e^{\int_{C} \ln f(x, y) d y} .
\end{aligned}
$$

Theorem 6. (Fundamental Theorem of Calculus for *line integral) Assume $D \subseteq \mathbb{R}^{2}$ is an open connected set and $C$ be a piecewise smooth simple curve in $D$. Assume $f$ is a continuously differentiable positive function on $D$. Then

$$
\int_{C} f_{x}^{*}(x, y)^{d x} f_{y}^{*}(x, y)^{d y}=\frac{f(x(b), y(b))}{f(x(a), y(a))},
$$

where $C=\{(x(t), y(t)): a \leq t \leq b\}$. 
Proof:

$$
\begin{aligned}
\int_{C} f_{x}^{*}(x, y)^{d x} f_{y}^{*}(x, y)^{d y} & =\int_{C} f_{x}^{*}(x, y)^{d x} \int_{C} f_{y}^{*}(x, y)^{d y} \\
& =e^{\int_{C} \ln f_{x}^{*}(x, y) d x} \cdot e^{\int_{C} \ln f_{y}^{*}(x, y) d y} \\
& =e^{\int_{C} \ln e^{\frac{f_{x}^{\prime}(x, y)}{f(x, y)}} d x} \cdot e^{\int_{C} \ln e^{\frac{f_{y}^{\prime}(x, y)}{f(x, y)}} d y} \\
& =e^{\int_{C}(\ln f(x, y))_{x}^{\prime} d x} \cdot e^{\int_{C}(\ln f(x, y))_{y}^{\prime} d y} \\
& =e^{\int_{C}(\ln f(x, y))_{x}^{\prime} d x+(\ln f(x, y))_{y}^{\prime} d y} \\
& =\frac{f(x(b), y(b))}{f(x(a), y(a))}
\end{aligned}
$$

which proves the theorem.

\subsubsection{Complex *integral}

Let $f(z)$ be a continuous nowhere-vanishing complex-valued function and let $z(t)=$ $x(t)+i y(t), a \leq t \leq b$. Assume $C$ is a piecewise smooth simple curve.To define $*$ integral of $f$ we need to represent $\log f$ as composition of branches of $\log$ and $f$ along $C$, to do that, we use method of localization and we consider the length of $[a, b]$ to be sufficiently small so that all values of $f(z(t))$ fall into an open half plane bounded by a line passing through origin, using this assumption we restrict $\log f$ to be as composition of branches of $\log$ and $f$. Now, let us construct the complex version of *integral. Consider $\mathscr{P}=\left\{z_{0}, \ldots, z_{m}\right\}$ be a partition of $C$. The integral product can be written as :

$$
\begin{aligned}
\prod_{k=1}^{m} e^{\Delta z_{k} \log f\left(\zeta_{k}\right)} & =e^{\sum_{k=1}^{m}\left(\mathscr{L}\left(f\left(\zeta_{k}\right)\right)+2 \pi n i\right) \Delta z_{k}} \\
& =e^{2 \pi n(z(b)-z(a)) i} e^{\sum_{k=1}^{m} \mathscr{L}\left(f\left(\zeta_{k}\right)\right) \Delta z_{k}}
\end{aligned}
$$

where

$$
\Delta z_{k}=z_{k}-z_{k-1}, n=0, \pm 1, \pm 2, \ldots
$$

and $\mathscr{L}$ is a branch of $\log$. Note that 


$$
P_{0}(f, \mathscr{P})=e^{\sum_{k=1}^{m} \mathscr{L}\left(f\left(\zeta_{k}\right)\right) \Delta z_{k}}
$$

and

$$
P_{n}(f, \mathscr{P})=e^{2 \pi n(z(b)-z(a)) i} P_{0}(f, \mathscr{P}), n=0, \pm 1, \pm 2, \ldots
$$

then, the limit of $P_{0}(f, \mathscr{P})$ when $\max \left\{\left|\Delta z_{1}\right|, \ldots,\left|\Delta z_{m}\right|\right\} \rightarrow 0$ is called a branch value of the complex *integral of $f$ along $C$ and it is denoted by $I_{0}^{*}(f, C)$, so, the complex *integral of $f$ is defined as

$$
I_{n}^{*}(f, C)=e^{2 \pi n(z(b)-z(a)) i} I_{0}^{*}(f, C), n=0, \pm 1, \pm 2, \ldots
$$

which is denoted by $\int_{C} f(z)^{d z}$.

Special Cases: Case 1: if $z(b)-z(a) \in \mathbb{Z}$ then $\int_{C} f(z)^{d z}=I_{0}^{*}(f, C)$ which is single valued. Case 2: if $z(b)-z(a)=q \in \mathbb{Q}\left(q=\frac{k}{p}\right.$ then, $\int_{C} f(z)^{d z}=e^{2 \pi n k i / p} I_{0}^{*}(f, C), n=$ $0,1, \ldots, p-1$ which has $p$ distinct values. Case 3 : if $z(b)-z(a)=r \in \mathbb{R}$ then for all $n$ we have $\left|I_{n}^{*}(f, C)\right|=\left|I_{0}^{*}(f, C)\right|$.

Proposition 1. The existence of the complex *integral can be reduced to the existence of line *integrals in the form below ;

$$
I_{n}^{*}(f, C)=\int_{C} R(z)^{d x}\left(e^{-\Theta(z)-2 \pi n}\right)^{d y} e^{i \ln \int_{C}\left(e^{\Theta(z)+2 \pi n}\right)^{d x} R(z)^{d y}} .
$$

Proof: Let $R(z)=|f(z)|, \Theta(z)=\operatorname{Im} \mathscr{L}(f(z))$ and $z=x+i y$ then:

$$
\begin{aligned}
P_{0}(f, \mathscr{P}) & =e^{\sum_{k=1}^{m} \mathscr{L}\left(f\left(\zeta_{k}\right)\right) \Delta z_{k}} \\
& =e^{\sum_{k=1}^{m}\left(\ln R\left(\zeta_{k}\right)+i \Theta\left(\zeta_{k}\right)\right)\left(\Delta x_{k}+i \Delta y_{k}\right)} \\
& =e^{\sum_{k=1}^{m}\left(\ln R\left(\zeta_{k}\right) \Delta x_{k}-\Theta\left(\zeta_{k}\right) \Delta y_{k}\right)+i \sum_{k=1}^{m}\left(\Theta\left(\zeta_{k}\right) \Delta x_{k}+\ln R\left(\zeta_{k}\right) \Delta y_{k}\right)} .
\end{aligned}
$$


Take limit of both sides as $\max \left\{\left|\Delta z_{1}\right|, \ldots,\left|\Delta z_{m}\right|\right\} \rightarrow 0$, then it is going to be line *integral denoted by

$$
I_{0}^{*}(f, C),
$$

and equal to

$$
e^{\int_{C}(\ln R(z) d x-\Theta(z) d y)+i \int_{C}(\Theta(z) d x+\ln R(z) d y)} .
$$

On the other hand, we know that

$$
I_{n}^{*}(f, C)=e^{2 \pi n(z(b)-z(a)) i} I_{0}^{*}(f, C),
$$

and

$$
e^{2 \pi n(z(b)-z(a)) i}=e^{2 \pi n(-(y(b)-y(a))+i(x(b)-x(a)))}=e^{-\int_{C} 2 \pi n d y+i \int_{C} 2 \pi n d x} .
$$

so,

$$
I_{n}^{*}(f, C)=e^{\int_{C}(\ln R(z) d x-(\Theta(z)+2 \pi n) d y)+i \int_{C}((\Theta(z)+2 \pi n) d x+\ln R(z) d y)}
$$

for $n=0, \pm 1, \pm 2, \ldots$. If we convert Newtonian Line integral to line *integral we will get what we were looking for. As it is stated at the beginning of this section, $f$ is nowhere vanishing and continuous function on open connected set $D$ and we use localization for what all values of $f(z(t)), a \leq t \leq b$ fall into an open half plane bounded by a line passing through origin, these conditions assures the existence of $\int_{C} f(z) d z$ as multiple values.

Theorem 7. (Local form of Fundamental Theorem of Complex *Calculus) Assume $f$ is a nowhere-vanishing *holomorphic function on an open connected set $D$ and $C=$ $\{z(t): a \leq t \leq b\}$ is a piecewise smooth simple curve in $D$ such that all values of $f(z(t))$ fall into an open half plane bounded by a line passing through origin. Then

$$
\int_{C} f^{*}(z)^{d z}=\left\{e^{2 \pi n(z(b)-z(a)) i} f(z(b)) / f(z(a)): n=0, \pm 1, \pm 2, \ldots\right\} .
$$


Proof: By using proposition 1,

$$
\int_{C} f^{*}(z)^{d z}=\int_{C}\left|f^{*}(z)\right|^{d x}\left(e^{-\arg f^{*}(z)}\right)^{d y} e^{i \ln \int_{C}\left(e^{\arg f^{*}(z)}\right)^{d x}\left|f^{*}(z)\right|^{d y}} .
$$

and Using Lemma 4, we have,

$$
\begin{aligned}
I_{n}^{*}\left(f^{*}, C\right)= & \int_{C} R_{x}^{*}(z)^{d x}\left(e^{[\ln R]_{y}^{\prime}(z)-2 \pi n}\right)^{d y} e^{i \ln \int_{C}\left(e^{\Theta_{x}^{\prime}(z)+2 \pi n}\right)^{d x}\left[e^{\Theta}\right]_{y}^{*}(z)^{d y}} \\
= & \int_{C} R_{x}^{*}(z)^{d x} R_{y}^{*}(z)^{d y} e^{i \ln \int_{C}\left[e^{\Theta}\right]_{x}^{*}(z)^{d x}\left[e^{\Theta}\right]_{y}^{*}(z)^{d y}} \\
& \times \int_{C}\left(e^{-2 \pi n}\right)^{d y} e^{i \ln \int_{C}\left(e^{2 \pi n}\right)^{d x}} .
\end{aligned}
$$

Using Fundamental Theorem of Calculus for *line integral we get that,

$$
\int_{C} R_{x}^{*}(z)^{d x} R_{y}^{*}(z)^{d y} e^{i \ln \int_{C}\left[e^{\Theta}\right]_{x}^{*}(z)^{d x}\left[e^{\Theta}\right]_{y}^{*}(z)^{d y}}=\frac{R(z(b)) e^{i \Theta(z(b))}}{R(z(a)) e^{i \Theta(z(a))}}=\frac{f(z(b))}{f(z(a))},
$$

and also we have,

$$
\int_{C}\left(e^{-2 \pi n}\right)^{d y} e^{i \ln \int_{C}\left(e^{2 \pi n}\right)^{d x}}=e^{2 \pi n(-(y(b)+y(a))+i(x(b)-x(a)))}=e^{2 \pi n(z(b)-z(a)) i} .
$$

which proves our aim. Now, we are in the position of constructing complex *integral in general case. To construct it we will be in need of lemma below;

Lemma 7. Assume $f$ is nowhere-vanishing continuous function on an open connected set $D$ and $C=\{z(t): a \leq t \leq b\}$ is a piecewise smooth simple curve in $D$. Then there exists a partition $\mathscr{P}=\left\{t_{0}, t_{1}, \ldots, t_{m}\right\}$ of $[a, b]$ such that each of the sets $\left\{f(z(t)): t_{k-1} \leq\right.$ $\left.t \leq t_{k}\right\}, k=1, \ldots, m$, falls into an open half plane bounded by a line through origin.

Proof: Let $t \in[a, b]$ and $\theta_{t}=\operatorname{Arg} f(z(t))$ and $\forall t \in(a, b)$ consider $L_{t}$ be the line made by $\theta_{t}+\pi / 2$ and $\theta_{t}-\pi / 2$. Since $f$ is continuous and nowhere-vanishing so,

$$
\exists\left(t-\varepsilon_{t}, t+\varepsilon_{t}\right) \subseteq[a, b]
$$

such that

$$
\left\{f(z(s)): t-\varepsilon_{t}<s<t+\varepsilon_{t}\right\}
$$

is a subset of open half planes bounded by $L_{t}$. 
Now, for $t=a$ consider $\left[a, a+\varepsilon_{a}\right)$ and for $t=b$ consider $\left(b-\varepsilon_{b}, b\right]$ then the collection of all such intervals makes an open cover of $[a, b] \subseteq \mathbb{R}$.If we consider the endpoints of these intervals in increasing order $a=t_{0}<t_{1}<\cdots<t_{m}=b$ we can get the required partition.

Now, to construct general form of complex *integral we follow these steps: consider $\mathscr{P}=\left\{t_{0}, t_{1}, \ldots, t_{m}\right\}$ be a partition of $[a, b]$ mentioned in previous Lemma and

$$
C_{k}=\left\{z(t): t_{k-1} \leq t \leq t_{k}\right\}
$$

Select branch $\mathscr{L}_{1}$ of log and let $\int_{C_{1}} f(z)^{d z}$ as defined in the local form. Then select a branch $\mathscr{L}_{2}$ of $\log$ such that

$$
\mathscr{L}_{2}\left(f\left(z\left(t_{1}\right)\right)\right)=\mathscr{L}_{1}\left(f\left(z\left(t_{1}\right)\right)\right)
$$

and define $\int_{C_{2}} f(z)^{d z}$. Next, select branch $\mathscr{L}_{3}$ of log such that

$$
\mathscr{L}_{3}\left(f\left(z\left(t_{2}\right)\right)\right)=\mathscr{L}_{2}\left(f\left(z\left(t_{2}\right)\right)\right)
$$

and define $\int_{C_{3}} f(z)^{d z}$, continue in this way to find $\mathscr{L}_{4}, \mathscr{L}_{5}, \ldots, \mathscr{L}_{m}$ and define $\int_{C_{4}} f(z)^{d z}$, $\int_{C_{5}} f(z)^{d z}, \ldots, \int_{C_{m}} f(z)^{d z}$ Note that the selection of first branch $\mathscr{L}_{1}$ is free, but the others must be selected to construct a continuous single-valued function $g$ on $[a, b]$ such that $g(t)$ at fixed $t \in[a, b]$ is equal to one of the branch values of $\log f(z(t))$. Now, following to Proposition 1,

$$
\begin{aligned}
I_{n}^{*}(f, C) & =\prod_{k=1}^{m} e^{2 \pi n\left(z\left(t_{k}\right)-z\left(t_{k-1}\right)\right) i+\int_{C_{k}} \mathscr{L}_{k}(f(z)) d z}, n=0, \pm 1, \pm 2, \ldots \\
& =e^{2 \pi n(z(b)-z(a)) i} e^{\sum_{k=1}^{m} \int_{C_{k}} \mathscr{L}_{k}(f(z)) d z}
\end{aligned}
$$




\subsubsection{Properties of Complex *Integral}

Let $f$ and $g$ be nowhere vanishing continuous functions on open connected set $D$ and $C=\{z(t)=x(t)+i y(t): a \leq t \leq b\}$ be a piecewise smooth simple curve in $D$. The properties of complex *integral are as follows;

(a) $\int_{C} f(z)^{d z}=\int_{C_{1}} f(z)^{d z} \int_{C_{2}} f(z)^{d z}$

where

$C_{1}=\{z(t)=x(t)+i y(t): a \leq t \leq c\}$

and

$C_{2}=\{z(t)=x(t)+i y(t): c \leq t \leq b\}$.

(b) $\int_{C}(f(z) g(z))^{d z}=\int_{C} f(z)^{d z} \int_{C} g(z)^{d z}$

(c) $\int_{C}(f(z) / g(z))^{d z}=\int_{C} f(z)^{d z} / \int_{C} g(z)^{d z}$

(d) $\int_{C} f(z)^{d z}=\left(\int_{-C} f(z)^{d z}\right)^{-1}$

(e) $\left(\int_{C} f(z)^{d z}\right)^{n} \subseteq \int_{C}\left(f(z)^{n}\right)^{d z}$.

\section{Proof:}

(a) We know that,

$$
I_{n}^{*}(f, C)=e^{2 \pi n(z(b)-z(a)) i} e^{\sum_{k=1}^{m} \int_{C_{k}} \mathscr{L}_{k}(f(z)) d z}
$$

where

$$
C_{k}=C_{1 k}+C_{2 k}
$$

then;

$$
\begin{aligned}
I_{n}^{*}(f, C) & =e^{2 \pi n(z(b)-z(a)) i} e^{\sum_{k=1}^{m} \int_{C_{1 k}+C_{2 k}} \mathscr{L}_{k}(f(z)) d z} \\
& =e^{2 \pi n(z(b)-z(a)) i} e^{\left.\sum_{k=1}^{m} \iint_{C_{1 k}} \mathscr{L}_{k}(f(z)) d z+\int_{C_{2 k}} \mathscr{L}_{k}(f(z)) d z\right]} \\
& =e^{2 \pi n(z(b)-z(a)) i} e^{\sum_{k=1}^{m} \int_{C_{1 k}} \mathscr{L}_{k}(f(z)) d z} e^{\sum_{k=1}^{m} \int_{C_{2 k}} \mathscr{L}_{k}(f(z)) d z} \\
& =I_{n}^{*}\left(f, C_{1}\right) I_{n}^{*}\left(f, C_{2}\right) .
\end{aligned}
$$


(b) To prove part (b) we will do process below,

$$
\begin{aligned}
I_{n}^{*}(f g, C) & =e^{2 \pi n(z(b)-z(a)) i} e^{\sum_{k=1}^{m} \int_{C_{k}} \mathscr{L}_{k}(f(z) g(z)) d z} \\
& =e^{2 \pi n(z(b)-z(a)) i} e^{\sum_{k=1}^{m} \int_{C_{k}}\left(\mathscr{L}_{k}(f(z))+\mathscr{L}_{k}(f(z))\right) d z} \\
& =e^{2 \pi n(z(b)-z(a)) i} e^{\sum_{k=1}^{m} \int_{C_{k}} \mathscr{L}_{k}(f(z)) d z} e^{\sum_{k=1}^{m} \int_{C_{k}} \mathscr{L}_{k}(g(z)) d z} \\
& =I_{n}^{*}(f, C) I_{n}^{*}(g, C) .
\end{aligned}
$$

(c) This part can be shown as,

$$
\begin{aligned}
I_{n}^{*}(f / g, C) & =e^{2 \pi n(z(b)-z(a)) i} e^{\sum_{k=1}^{m} \int_{C_{k}} \mathscr{L}_{k}(f(z) / g(z)) d z} \\
& =e^{2 \pi n(z(b)-z(a)) i} e^{\sum_{k=1}^{m} \int_{C_{k}}\left(\mathscr{L}_{k}(f(z))-\mathscr{L}_{k}(f(z))\right) d z} \\
& =e^{2 \pi n(z(b)-z(a)) i} \frac{e^{\sum_{k=1}^{m} \int_{C_{k}} \mathscr{L}_{k}(f(z)) d z}}{e^{\sum_{k=1}^{m} \int_{C_{k}} \mathscr{L}_{k}(g(z)) d z}} \\
& =I_{n}^{*}(f, C) / I_{n}^{*}(g, C) .
\end{aligned}
$$

(d) Same as previous parts the proof of this part can be fulfilled as;

$$
\begin{aligned}
I_{n}^{*}(f,-C) & =e^{2 \pi n(z(b)-z(a)) i} e^{\sum_{k=1}^{m} \int_{-C_{k}} \mathscr{L}_{k}(f(z)) d z} \\
& =e^{2 \pi n(z(b)-z(a)) i} e^{\sum_{k=1}^{m}\left(\int_{C_{k}} \mathscr{L}_{k}(f(z)) d z\right)^{-1}} \\
& =\left(\int_{-C} f(z)^{d z}\right)^{-1} .
\end{aligned}
$$

(e) We know that

$$
A^{n} \subseteq A A \cdots A
$$

where

$$
A^{n}=\left\{a^{n}: a \in A\right\}
$$

and

$$
A A \cdots A(n \text { times })=\left\{a_{1} a_{2} \cdots a_{n}: a_{i} \in A, i=1, \ldots, n\right\} .
$$


Now, let

$$
A=\int_{C} f(z)^{d z}
$$

then by using property $(b)$ we have,

$$
\left(\int_{C} f(z)^{d z}\right)^{n} \subseteq \int_{C} f(z)^{d z} \int_{C} f(z)^{d z} \ldots \int_{C} f(z)^{d z}
$$

and also we know that

$$
\int_{C} f(z)^{d z} \int_{C} f(z)^{d z} \ldots \int_{C} f(z)^{d z}=\int_{C}(f(z) f(z) \ldots f(z))^{d z}=\int_{C}\left((f(z))^{n}\right)^{d z}
$$

At the end of this section, to show the application of our result we bring some examples.

Example 1. It is already shown that $f^{*}(z)=e^{c}$ where $f(z)=e^{c z}, z \in \mathbb{C}$ and $c=$ const $\in \mathbb{C}$,so,

$$
\int_{C}\left(e^{c}\right)^{d z}=e^{2 \pi n(z(b)-z(a)) i} e^{c(z(b)-z(a))}=e^{(z(b)-z(a))(c+2 \pi n i)},
$$

where $C=\{z(t): a \leq t \leq b\}$ is a piecewise smooth curve.

Example 2. Let $f(z)=e^{c e^{z}}, z \in \mathbb{C}$, where $c=$ const $\in \mathbb{C}$. It has been established that $f^{*}(z)=f(z)$. Consequently,

$$
\int_{C}\left(e^{c e^{z}}\right)^{d z}=e^{2 \pi n(z(b)-z(a)) i} e^{c\left(e^{z(b)}-e^{z(a)}\right)} .
$$

Example 3. Let us evaluate the analog of

$$
\oint_{|z|=1} \frac{d z}{z}=2 \pi i
$$

in complex *calculus. Assuming that the orientation on the unit circle $|z|=1$ is positive, 


$$
\oint_{|z|=1}\left(e^{1 / z}\right)^{d z}=e^{\oint_{|z|=1} \log e^{1 / z} d z}=e^{\oint_{|z|=1}\left(\frac{1}{z}+2 \pi n i\right) d z}=e^{2 \pi n(z(b)-z(a)) i} e^{2 \pi i}=1 .
$$

which shows that the *integral over piecewise smooth simple closed curve of $f^{*}$ is 1. This demonstrates that, complex *integral does not count residues and therefore, it needs further improvements. 


\section{Chapter 4}

\section{RIEMANN SURFACE OF COMPLEX LOGARITHM}

In the previous chapter we constructed multiplicative version of derivative and integral in complex case. Due to the capacity of complex space $\mathbb{C}$, we could define *derivative and its properties well but we proved that $*$ integral is multi-valued, because of multi-valued nature of Logarithm, with complicated properties and also we found that *integral does not count residues and singularities. In the chapter forward, we want to show that this drawback can be solved if we modify complex space $\mathbb{C}$ and define generalized complex space denoted by $\mathbb{B}$, since $\mathbb{C}$ is not sufficient for our purpose and this is why we will use Riemann surface of complex Logarithm function. This modification also solves the multi-valued nature of logarithm.

In most of textbooks related to complex variables and applications [4, 5, 6] the differentiation and integration of complex functions are on the base of algebraic form of complex variables and the polar form is used rarely. In this chapter derivatives and integrals are represented on the base of polar form of complex variables on $\mathbb{B}$ and we will show that if we transfer the sense of complex integral and complex derivative to Bi-geometric then the polar representation of complex functions on $\mathbb{B}$ is going to be as the same as algebraic form on $\mathbb{C}$. The organization of this chapter is as follows; First, Bi-geometric differentiation and integration will be reviewed on real case then after defining $\mathbb{B}$ and properties of $\mathbb{B}$ we will construct analogue of complex functions and variables in bigeometric case and finally, integration and differentiation of bigeometric complex functions will be performed on $\mathbb{B}$. 


\subsection{Bi-geometric Real Calculus}

Let $\alpha: \mathbb{R} \rightarrow \mathbb{I} \subset \mathbb{R}$ be a bijective function. To construct $\alpha$-calculus, first of all we have to transfer field properties of $\mathbb{R}$ to $\mathbb{I} \subset \mathbb{R}$ as;

(i) $a \oplus_{\alpha} b=\alpha\left(\alpha^{-1}(a)+\alpha^{-1}(b)\right)$,

(ii) $a \otimes_{\alpha} b=\alpha\left(\alpha^{-1}(a) \times \alpha^{-1}(b)\right)$,

(iii) $a \ominus_{\alpha} b=\alpha\left(\alpha^{-1}(a)-\alpha^{-1}(b)\right)$,

(iv) $a \oslash_{\alpha} b=\alpha\left(\alpha^{-1}(a) / \alpha^{-1}(b)\right)$.

which are called $\alpha$-addition, $\alpha$-multiplication, $\alpha$-subtraction and $\alpha$-division respectively. Based on $\alpha$-operations, $\alpha$-derivative and $\alpha$-integral can be defined as follows,

$$
\lim _{m e s h \rightarrow 0}\left(f(x) \ominus_{\alpha} f\left(x_{0}\right) \oslash_{\alpha}\left(x \ominus_{\alpha} x_{0}\right)\right.
$$

and

$$
\lim _{m e s h \rightarrow 0} \bigoplus_{i=1}^{n} \alpha f\left(c_{i}\right) \otimes_{\alpha}\left(x_{i} \ominus_{\alpha} x_{i-1}\right)
$$

All of these calculi are isometric, for example the most popular non-newtonian calculus is based on exponential functions $\alpha(x)=e^{x}$. In this case $\alpha$-operations can be defined as:

(i) $a \oplus_{\exp } b=e^{\ln a+\ln b}=a b$,

(ii) $a \otimes_{\exp } b=e^{\ln a \ln b}=a^{\ln b}=b^{\ln a}$,

(iii) $a \ominus_{\exp } b=e^{\ln a-\ln b}=a / b$,

(iv) $a \oslash_{\exp } b=e^{\ln a / \ln b}=a^{1 / \ln b}$.

which define a field on $\mathbb{I}=(0, \infty)$ of exponential function with neutral element of expaddition and exp-multiplication 1 and e, respectively. As it is already mentioned, on the 
base of $\alpha$-operations, $\alpha$-derivative and integral can be constructed. Now, if $\alpha(x)=e^{x}$ then exp-derivative and exp-integral will be defined as follows;

$$
\begin{aligned}
\left(f(y) \ominus_{\exp } f(x)\right) \oslash_{\exp }\left(y \ominus_{\exp } x\right) & =(f(y) / f(x))^{\frac{1}{\ln (y / x)}} \\
& =(f(y) / f(x))^{\frac{1}{y-x} \cdot \frac{y-x}{\ln y-\ln x}} \\
& =\left(e^{\ln \left(\frac{f(y)}{f(x)}\right)}\right)^{\frac{1}{y-x} \cdot \frac{y-x}{\ln y-\ln x}} \\
& =\left(e^{\frac{\ln f(y)-\ln f(x)}{y-x}}\right)^{\frac{y-x}{\ln y-\ln x}} \\
& \rightarrow e^{\frac{(\ln f(x))^{\prime}}{(\ln x)^{\prime}}}=e^{x(\ln f(x))^{\prime}},
\end{aligned}
$$

and

$$
\begin{aligned}
\bigoplus_{i=1}^{n} \exp f\left(c_{i}\right) \otimes_{\exp }\left(x_{i} \ominus_{\exp } x_{i-1}\right) & =\prod_{i=1}^{n} f\left(c_{i}\right)^{\ln \left(x_{i} / x_{i-1}\right)} \\
& =e^{\sum_{i=1}^{n} \ln f\left(c_{i}\right)\left(x_{i}-x_{i-1}\right) \cdot \frac{\ln x_{i}-\ln x_{i-1}}{x_{i}-x_{i-1}}} \\
& \rightarrow e^{\int_{a}^{b} \ln f(x)(\ln x)^{\prime} d x}=e^{\int_{a}^{b} \frac{\ln f(x)}{x} d x} .
\end{aligned}
$$

There are two different calculi defined on the base of exp-operations, Multiplicative calculus, which was demonstrated in the previous chapters and Bi-geometric calculus. Bi-geometric derivative and Bi-geometric integral, or briefly $\pi_{\text {derivative and }} \pi_{\text {integral }}$ are defined by

$$
f^{\pi}(x)=e^{x(\ln f(x))^{\prime}}
$$

and

$$
\int_{a}^{b} f(x)^{\mathbf{d} x}=e^{\int_{a}^{b} \frac{\ln f(x)}{x} d x}
$$

These two non-Newtonian calculi are suitable for growth problems that were used and investigated in [3],[4],[8] and [28]. 


\subsection{The Field $\mathbb{B}$}

In this section, we will show that the mentioned drawbacks of *integral can be removed if we use field $\mathbb{B}$ and $\pi$-operations.

Definition 8. The algebraic form of Riemann surface of complex logarithm is $\mathbb{B}=$ $\{(r, \theta): r>0,-\infty<\theta<\infty\}$ where $z=r e^{i \theta} \in \mathbb{C}$ and $\theta$ is its principal argument.

Note: The subset $\mathbb{B}_{\alpha}=\{(r, \theta): r>0, \alpha-\pi \leq \theta<\alpha+\pi\}$ of $\mathbb{B}$ is called $\alpha$-branch of $\mathbb{B}$.

Identification:The identity branch of $\mathbb{B}$ is denoted by $\mathbb{B}_{0}$ and defined as $\mathbb{B}_{0} \ni(r, \theta)=$ $r e^{i \theta} \in \mathbb{C} \backslash\{0\}$

Notification: To recognize elements of $\mathbb{B}$ and $\mathbb{C}$ and functions belong $\mathbb{B}$ and $\mathbb{C}$, we will use notifications as follows: elements of $\mathbb{B}$ (b-number) are shown as boldface letter $\mathbf{z}$, functions with domain and range in $\mathbb{B}$ are denoted as $\mathbf{f}$, and functions and elements belonging to $\mathbb{C}$ are called c-number are shown as normal letters like $z$ and $f: \mathbb{C} \rightarrow \mathbb{C}$.

\subsubsection{Algebraic Operations on $\mathbb{B}$}

The multiplication and division operations on $\mathbb{B}$ can be defined as follows;

$$
\begin{gathered}
\mathbf{z}_{1} \mathbf{z}_{2}=\left(r_{1}, \theta_{1}\right)\left(r_{2}, \theta_{2}\right)=\left(r_{1} r_{2}, \theta_{1}+\theta_{2}\right) \\
\mathbf{z}_{1} / \mathbf{z}_{2}=\left(r_{1}, \theta_{1}\right) /\left(r_{2}, \theta_{2}\right)=\left(r_{1} / r_{2}, \theta_{1}-\theta_{2}\right) .
\end{gathered}
$$

To define exp-multiplication $\left(\otimes_{\mathbf{e x p}}\right)$ and $\mathbf{e x p}$-division $\left(\oslash_{\mathbf{e x p}}\right)$ on $\mathbb{B}$ consider the lemma below. Before starting lemma, let us define two functions $\mathbf{E}^{z}(z \in \mathbb{C})$ and $\log \mathbf{z}(\mathbf{z} \in \mathbb{B})$ where $\mathbf{E}^{z}$ is a bijective version of exponential function from $\mathbb{C}$ to $\mathbb{B}$ defined as

$$
\mathbf{E}^{z}=\left(e^{x}, y\right), z=x+i y \in \mathbb{C}
$$

and

$$
\log \mathbf{z}=\ln r+i \theta, \mathbf{z}=(r, \theta) \in \mathbb{B}
$$


is the inverse of $\mathbf{E}^{z}$.

Lemma 8. The following hold.

(a) For $\mathbf{z}_{1}, \mathbf{z}_{2} \in \mathbb{B}, \log \left(\mathbf{z}_{1} \mathbf{z}_{2}\right)=\log \mathbf{z}_{1}+\log \mathbf{z}_{2}$, and $\log \left(\mathbf{z}_{1} / \mathbf{z}_{2}\right)=\log \mathbf{z}_{1}-\log \mathbf{z}_{2}$

(b) For $z_{1}, z_{2} \in \mathbb{C}, \mathbf{E}^{z_{1}+z_{2}}=\mathbf{E}^{z_{1}} \mathbf{E}^{z_{2}}$, and $\mathbf{E}^{z_{1}-z_{2}}=\mathbf{E}^{z_{1}} / \mathbf{E}^{z_{2}}$

(c) For $z \in \mathbb{C}, \log \mathbf{E}^{z}=z$.

(d) For $\mathbf{z} \in \mathbb{B}, \mathbf{E}^{\log \mathbf{z}}=\mathbf{z}$.

Proof: (a) Using definition of Log we have,

$$
\begin{aligned}
\log \left(\mathbf{z}_{1} \mathbf{z}_{2}\right) & =\log \left(\left(r_{1}, \theta_{1}\right),\left(r_{2}, \theta_{2}\right)\right) \\
& =\log \left(r_{1} r_{2}, \theta_{1}+\theta_{2}\right) \\
& =\ln \left(r_{1} r_{2}\right)+i\left(\theta_{1}+\theta_{2}\right) \\
& =\left(\ln r_{1}+\ln r_{2}\right)+i\left(\theta_{1}+\theta_{2}\right) \\
& =\left(\ln r_{1}+i \theta_{1}\right)+\left(\ln r_{2}+i \theta_{2}\right) \\
& =\log \mathbf{z}_{1}+\log \mathbf{z}_{2} .
\end{aligned}
$$

And the process is same to prove second part. 
(b) We have,

$$
\begin{aligned}
\mathbf{E}^{z_{1}+z_{2}} & =\mathbf{E}^{\left(x_{1}+i y_{1}\right)+\left(x_{2}+i y_{2}\right)} \\
& =\mathbf{E}^{\left(x_{1}+x_{2}\right)+i\left(y_{1}+y_{2}\right)} \\
& =\left(e^{x_{1}+x_{2}}, y_{1}+y_{2}\right) \\
& =\left(e^{x_{1}} e^{x_{2}}, y_{1}+y_{2}\right) \\
& =\left(e^{x_{1}}, y_{1}\right)\left(e^{x_{2}}, y_{2}\right) \\
& =\mathbf{E}^{z_{1}} \mathbf{E}^{z_{2}} .
\end{aligned}
$$

. (c) It can be shown that,

$$
\begin{aligned}
\log \mathbf{E}^{z} & =\log \mathbf{E}^{x+i y} \\
& =\log \left(e^{x}, y\right) \\
& =\ln \left(e^{x}\right)+i y \\
& =x+i y \\
& =z .
\end{aligned}
$$

(d) Clearly,

$$
\begin{aligned}
\mathbf{E}^{\mathbf{L o g} \mathbf{z}} & =\mathbf{E}^{\mathbf{L o g}(r, \theta)} \\
& =\mathbf{E}^{\ln r+i \theta} \\
& =\left(e^{\ln r}, \boldsymbol{\theta}\right) \\
& =(r, \theta) \\
& =\mathbf{z} .
\end{aligned}
$$

We will use the above lemma to define exp-multiplication and exp-division on $\mathbb{B}$ as

$$
\mathbf{z}_{1} \otimes_{\exp } \mathbf{z}_{2}=\mathbf{E}^{\log \mathbf{z}_{1} \log \mathbf{z}_{2}}=\left(e^{\ln r_{1} \ln r_{2}-\theta_{1} \theta_{2}}, \theta_{2} \ln r_{1}+\theta_{1} \ln r_{2}\right)
$$


and

$$
\mathbf{z}_{1} \oslash \exp \mathbf{z}_{2}=\mathbf{E}^{\mathbf{L o g} \mathbf{z}_{1} / \log \mathbf{z}_{2}}=\left(e^{\frac{\ln r_{1} \ln r_{2}+\theta_{1} \theta_{2}}{\ln ^{2} r_{2}+\theta_{2}^{2}}}, \frac{\theta_{1} \ln r_{2}-\theta_{2} \ln r_{1}}{\ln ^{2} r_{2}+\theta_{2}^{2}}\right)
$$

One can prove that $\mathbb{B}$ is a field using operations above with neutral elements of $\otimes$, $\mathbf{1}=(e, 0)$ and neutral element of $\oplus, \mathbf{0}=(1,0)$. Now, let us define the periodic extension of $\mathbf{E}^{z}$ to $\overline{\mathbb{B}}=\{(r, \theta): r \geq 0,-\infty<\theta<\infty\}$ and its multi-valued inverse as follows;

$$
\mathbf{e}^{\mathbf{z}}=\mathbf{e}^{(r, \theta)}=\left(e^{r \cos \theta}, r \sin \theta\right), \mathbf{z}=(r, \theta) \in \overline{\mathbb{B}}
$$

since for any $\mathbf{z}=(r, \theta) \in \overline{\mathbb{B}}$ we have $x=r \cos \theta$ and $y=r \sin \theta$.

And

$$
\log \mathbf{z}=\left(\sqrt{\ln ^{2} r+\theta^{2}}, \operatorname{atan} 2(\theta, \ln r)+2 \pi n\right), n=0, \pm 1, \ldots, \mathbf{z}=(r, \theta) \in \mathbb{B},
$$

since for any $\mathbf{z}=(r, \theta)$ we have

$$
\log (r, \theta)=\ln r+i \theta=R e^{i \Theta}
$$

and in this case

$$
R=\sqrt{\ln ^{2} r+\theta^{2}}, \Theta=\operatorname{atan} 2(\theta, \ln r) .
$$

Hereby, $\log \mathbf{z}$ is the principal branch of $\log \mathbf{z}$.

\subsubsection{Elementary Functions on $\mathbb{B}$}

In this section we will define ${ }^{\pi}$ analoge of elementary complex functions and will construct the relation between these functions and its Newtonian version. As it is stated before, $\mathbf{e}^{\mathbf{z}}$ and $\log \mathbf{z}$ are ${ }^{\pi}$ analoge of $e^{z}$ and $\log z$,respectively. Clearly we can prove that

$$
\mathbf{E}^{z}=\mathbf{E}^{e^{\mathbf{L o g} \mathbf{z}}} \text { for } \mathbf{z}=z \in \mathbb{B}_{0} \subset \mathbb{B} \text {. }
$$

Since $\mathbf{E}^{z}=\mathbf{E}^{x+i y}=\left(e^{x}, y\right)$ and

$$
\mathbf{E}^{e^{\mathbf{L} \log z}}=\mathbf{E}^{e^{l n r+i \theta}}=\mathbf{E}^{r e^{i \theta}}=\mathbf{E}^{x+i y}=\left(e^{x}, y\right) .
$$

If $f$ is a single valued complex function, then its $\pi_{\text {analoge is denoted by } \mathbf{f} \text { and defined }}$ by

$$
\mathbf{f}(\mathbf{z})=\mathbf{E}^{f(\log \mathbf{z})}, \text { for any } \mathbf{z} \in B \subseteq \mathbb{B}
$$


Now, this definition motivates us to write $\pi_{\text {trigonometric functions and }}{ }^{\text {power func- }}$ tion.

(a) $\cos \mathbf{z}=\left(e^{\cosh \theta \cos \ln r},-\sinh \theta \sin \ln r\right)$,

(b) $\sin \mathbf{z}=\left(e^{\cosh \theta \sin \ln r}, \sinh \theta \cos \ln r\right)$,

(c) $\cosh \mathbf{z}=\left(e^{\cos \theta \cosh \ln r}, \sin \theta \sinh \ln r\right)$,

(d) $\sinh \mathbf{z}=\left(e^{\cos \theta \sinh \ln r}, \sin \theta \cosh \ln r\right)$,

(e) $\mathbf{z}^{\mathbf{w}}=\mathbf{e}^{\mathbf{w} \otimes \log (\log \mathbf{z})}$.

Proof: (a) Let us find first part as follows;

$$
\begin{aligned}
\mathbf{c o s} \mathbf{Z} & =\mathbf{E}^{\frac{1}{2}\left(e^{i \mathbf{L o g} \mathbf{z}}+e^{-i \mathbf{L o g} \mathbf{z}}\right)} \\
& =\mathbf{E}^{\frac{1}{2}\left(e^{-\theta+i \ln r}+e^{\theta-i \ln r}\right)} \\
& =\mathbf{E}^{\frac{1}{2}\left(e^{-\theta}(\cos \ln r+i \sin \ln r)+e^{\theta}(\cos \ln r-i \sin \ln r)\right)} \\
& =\mathbf{E}^{\frac{e^{\theta}+e^{-\theta}}{2} \cos \ln r-i \frac{e^{\theta}-e^{-\theta}}{2}} \sin \ln r \\
& =\mathbf{E}^{\cosh \theta \cos \ln r-i \sinh \theta \sin \ln r} \\
& =\left(e^{\cosh \theta \cos \ln r},-\sinh \theta \sin \ln r\right) .
\end{aligned}
$$

(b) It can be shown that,

$$
\begin{aligned}
\sin \mathbf{z} & =\mathbf{E}^{\frac{1}{2 i}\left(e^{i \mathbf{L o g} \mathbf{z}}-e^{-i \mathbf{L o g} \mathbf{z}}\right)} \\
& =\mathbf{E}^{\frac{-i}{2}\left(e^{-\theta+i \ln r}-e^{\theta-i \ln r}\right)} \\
& =\mathbf{E}^{\frac{-i}{2}\left(e^{-\theta}(\cos \ln r+i \sin \ln r)-e^{\theta}(\cos \ln r-i \sin \ln r)\right)} \\
& =\mathbf{E}^{\frac{e^{\theta}+e^{-\theta}}{2}} \sin \ln r+i \frac{\theta^{\theta}-e^{-\theta}}{2} \cos \ln r \\
& =\mathbf{E}^{\cosh \theta \sin \ln r+i \sinh \theta \cos \ln r} \\
& =\left(e^{\cosh \theta \sin \ln r}, \sinh \theta \cos \ln r\right) .
\end{aligned}
$$


(c) It follows from ;

$$
\begin{aligned}
& \cosh \mathbf{z}=\mathbf{E}^{\frac{1}{2}\left(e^{\log \mathbf{z}}+e^{-\mathbf{L o g} \mathbf{z}}\right)} \\
& =\mathbf{E}^{\frac{1}{2}\left(e^{\theta+i \ln r}+e^{-\theta-i \ln r}\right)} \\
& =\mathbf{E}^{\frac{1}{2}\left(e^{\ln r} e^{i \theta}+e^{-l n r} e^{-i \theta}\right)} \\
& =\mathbf{E}^{\frac{1}{2}\left(e^{\ln r}(\cos \theta+i \sin \theta)+e^{-l n r}(\cos \theta-i \sin \theta)\right)} \\
& =\mathbf{E}^{\frac{1}{2}\left(e^{l n r}+e^{-l n r}\right) \cos \theta+i\left(e^{\ln r}-e^{-l n r}\right) \sin \theta} \\
& =\mathbf{E}^{\cos \theta \cosh \ln r+i \sin \theta \sinh \ln r} \\
& =\left(e^{\cos \theta \cosh \ln r}, \sin \theta \sinh \ln r\right) .
\end{aligned}
$$

(d) We have;

$$
\begin{aligned}
& \sinh \mathbf{z}=\mathbf{E}^{\frac{1}{2}\left(e^{\mathbf{L o g} \mathbf{z}}-e^{-\mathbf{L o g} \mathbf{z}}\right)} \\
& =\mathbf{E}^{\frac{1}{2}\left(e^{\theta+i \ln r}-e^{-\theta-i \ln r}\right)} \\
& =\mathbf{E}^{\frac{1}{2}\left(e^{\ln r} e^{i \theta}-e^{-l n r} e^{-i \theta}\right)} \\
& =\mathbf{E}^{\frac{1}{2}\left(e^{\ln r}(\cos \theta+i \sin \theta)-e^{-\ln r}(\cos \theta-i \sin \theta)\right)} \\
& =\mathbf{E}^{\frac{1}{2}\left(e^{l n r}-e^{-l n r}\right) \cos \theta+i\left(e^{\ln r}+e^{-l n r}\right) \sin \theta} \\
& =\mathbf{E}^{\cos \theta \sinh \ln r+i \sin \theta \cosh \ln r} \\
& =\left(e^{\cos \theta \cosh \ln r}, \sin \theta \cosh \ln r\right) .
\end{aligned}
$$

(e) We know that $\mathbf{f}(\mathbf{z})=\mathbf{E}^{f(\log z)}$, for any $(z) \in \mathbb{B}$ and also $z^{w}$ for $z \in \mathbb{C}$ and $w \in \mathbb{C}$ can be written as $z^{w}=e^{w \log z}$ and its $\pi$ analoge is going to be as

$$
\mathbf{z}^{\mathbf{w}}=\mathbf{e}^{\mathbf{w} \otimes \log (\log \mathbf{z})}
$$

which proves part (e). 


\section{3 ${ }^{\pi}$ Derivative}

Let us start the following section by lemma below that relates to Cauchy-Riemann condition in ${ }^{\pi}$ calculus.

Lemma 9. Assume $f$ is non-vanishing function from a non-empty open connected subset $C$ of $\mathbb{C}$ to $\mathbb{C}$ with representations

$$
f(z)=u(r, \theta)+i v(r, \theta)=R(r, \theta) e^{i \Theta(r, \theta)} \text { for } z=r e^{i \theta} .
$$

If $f^{\prime}(z)$ exists and the Cauchy-Riemann conditions in polar form

$$
r u_{r}^{\prime}=v_{\theta}^{\prime} \text { and } r v_{r}^{\prime}=-u_{\theta}^{\prime}
$$

hold, then

$$
r(\ln R)_{r}^{\prime}=\Theta_{\theta}^{\prime} \text { and } r \Theta_{r}^{\prime}=-(\ln R)_{\theta}^{\prime}
$$

and conseuently

$$
z(\log f(z))^{\prime}=r\left((\ln R)_{r}^{\prime}+i \Theta_{r}^{\prime}\right)
$$

Proof: We know that $R=R(r, \theta)=\sqrt{u^{2}(r, \theta)+v^{2}(r, \theta)}$ and $\Theta(r, \theta)=\arctan (v(r, \theta), u(r, \theta))$,

so

$$
\begin{gathered}
R_{r}^{\prime}=\frac{u u_{r}^{\prime}+v v_{r}^{\prime}}{\sqrt{u^{2}+v^{2}}}=\frac{u u_{r}^{\prime}+v v_{r}^{\prime}}{R}, \\
R_{\theta}^{\prime}=\frac{u u_{\theta}^{\prime}+v v_{\theta}^{\prime}}{\sqrt{u^{2}+v^{2}}}=\frac{u u_{\theta}^{\prime}+v v_{\theta}^{\prime}}{R}, \\
\Theta_{r}^{\prime}=\frac{-v u_{r}^{\prime}}{u^{2}+v^{2}}+\frac{u v_{r}^{\prime}}{u^{2}+v^{2}}=\frac{u v_{r}^{\prime}-v u_{r}^{\prime}}{R^{2}},
\end{gathered}
$$

and

$$
\Theta_{\theta}^{\prime}=\frac{-v u_{\theta}^{\prime}}{u^{2}+v^{2}}+\frac{u v_{\theta}^{\prime}}{u^{2}+v^{2}}=\frac{u v_{\theta}^{\prime}-v u_{\theta}^{\prime}}{R^{2}} .
$$

So, by the Cauchy-Riemann conditions in polar form,

$$
r R_{r}^{\prime}=\frac{r\left(u u_{r}^{\prime}+v v_{r}^{\prime}\right)}{R}=\frac{u v_{\theta}^{\prime}-v u_{\theta}^{\prime}}{R}=R \Theta_{\theta}^{\prime} \Rightarrow r(\ln R)_{r}^{\prime}=\Theta_{\theta}^{\prime}
$$

and

$$
R_{\theta}^{\prime}=\frac{u u_{\theta}^{\prime}+v v_{\theta}^{\prime}}{R}=\frac{-r\left(u v_{r}^{\prime}-v u_{r}^{\prime}\right)}{R}=-r R \Theta_{r}^{\prime} \Rightarrow(\ln R)_{\theta}^{\prime}=-r \Theta_{r}^{\prime}
$$


which proves(10).

Now, to prove (2) we follow the steps below;

Clearly,

$$
f^{\prime}(z)=u_{x}^{\prime}+i v_{x}^{\prime}
$$

where

$$
u=u(r, \theta), v=v(r, \theta), r=\sqrt{x^{2}+y^{2}} \text { and } \theta=\tan ^{-1} \frac{y}{x},
$$

then by using chain rule we have

$$
\begin{aligned}
u_{x}^{\prime} & =u_{r}^{\prime} r_{x}^{\prime}+u_{\theta}^{\prime} \theta_{x}^{\prime} \\
& =u_{r}^{\prime} \frac{x}{\sqrt{x^{2}+y^{2}}}+u_{\theta}^{\prime} \frac{-y}{x^{2}+y^{2}} \\
& =u_{r}^{\prime} \frac{r \cos \theta}{r}+u_{\theta}^{\prime} \frac{-r \sin \theta}{r^{2}} \\
& =u_{r}^{\prime} \cos \theta-\frac{1}{r} u_{\theta}^{\prime} \sin \theta \\
& =u_{r}^{\prime} \cos \theta-\frac{1}{r}\left(-r v_{r}^{\prime}\right) \sin \theta \\
& =u_{r}^{\prime} \cos \theta+v_{r}^{\prime} \sin \theta .
\end{aligned}
$$

And similarly,

$$
v_{x}^{\prime}=v_{r}^{\prime} r_{x}^{\prime}+v_{\theta}^{\prime} \theta_{x}^{\prime}=v_{r}^{\prime} \cos \theta-u_{r}^{\prime} \sin \theta
$$

So,

$$
\begin{aligned}
f^{\prime}(z) & =u_{x}^{\prime}+i v_{x}^{\prime} \\
& =\left(u_{r}^{\prime}+i v_{r}^{\prime}\right) \cos \theta+\left(v_{r}^{\prime}-i u_{r}^{\prime}\right) \sin \theta \\
& =e^{-i \theta}\left(u_{r}^{\prime}+i v_{r}^{\prime}\right)
\end{aligned}
$$

Next, we have

$$
\left[\begin{array}{c}
R_{r}^{\prime} R \\
\Theta_{r}^{\prime} R^{2}
\end{array}\right]=\left[\begin{array}{cc}
u & v \\
-v & u
\end{array}\right]\left[\begin{array}{c}
u_{r}^{\prime} \\
v_{r}^{\prime}
\end{array}\right] .
$$


Which implies

$$
\left[\begin{array}{c}
u_{r}^{\prime} \\
v_{r}^{\prime}
\end{array}\right]=\frac{1}{R^{2}}\left[\begin{array}{cc}
u & -v \\
v & u
\end{array}\right]\left[\begin{array}{c}
R_{r}^{\prime} R \\
\Theta_{r}^{\prime} R^{2}
\end{array}\right] .
$$

Therefore,

$$
\begin{aligned}
f^{\prime}(z) & =e^{-i \theta}\left(u_{r}^{\prime}+i v_{r}^{\prime}\right)=e^{-i \theta}\left(\frac{R_{r}^{\prime}(u+i v)}{R}+i \Theta_{r}^{\prime}(u+i v)\right) \\
& =e^{-i \theta}(u+i v)\left(\frac{R_{r}^{\prime}}{R}+i \Theta_{r}^{\prime}\right)=e^{-i \theta} R e^{i \Theta}\left(\frac{R_{r}^{\prime}}{R}+i \Theta_{r}^{\prime}\right) \\
& =e^{i(\Theta-\theta)}\left(R_{r}^{\prime}+i R \Theta_{r}^{\prime}\right) .
\end{aligned}
$$

Then

$$
\begin{aligned}
(\log f(z))^{\prime} & =\frac{f^{\prime}(z)}{f(z)}=\frac{e^{i(\Theta-\theta)}\left(R_{r}^{\prime}+i R \Theta_{r}^{\prime}\right)}{\operatorname{Re}^{i \Theta}} \\
& =e^{-i \theta}\left((\ln R)_{r}^{\prime}+i \Theta_{r}^{\prime}\right)=(\cos \theta-i \sin \theta)\left((\ln R)_{r}^{\prime}+i \Theta_{r}^{\prime}\right) \\
& =\left((\ln R)_{r}^{\prime} \cos \theta+\Theta_{r}^{\prime} \sin \theta\right)+i\left(\Theta_{r}^{\prime} \cos \theta-(\ln R)_{r}^{\prime} \sin \theta\right)
\end{aligned}
$$

Consequently,

$$
\begin{aligned}
z(\log f(z))^{\prime}= & r(\cos \theta+i \sin \theta)\left(\left((\ln R)_{r}^{\prime} \cos \theta+\Theta_{r}^{\prime} \sin \theta\right)\right. \\
& \left.+i\left(\Theta_{r}^{\prime} \cos \theta-(\ln R)_{r}^{\prime} \sin \theta\right)\right)
\end{aligned}
$$

Here

$$
\begin{aligned}
\operatorname{Re}\left(z(\log f(z))^{\prime}\right)= & r\left((\ln R)_{r}^{\prime} \cos ^{2} \theta+\Theta_{r}^{\prime} \sin \theta \cos \theta\right. \\
& \left.-\Theta_{r}^{\prime} \sin \theta \cos \theta+(\ln R)_{r}^{\prime} \sin ^{2} \theta\right) \\
= & r(\ln R)_{r}^{\prime},
\end{aligned}
$$

and 


$$
\begin{aligned}
\operatorname{Im}\left(z(\log f(z))^{\prime}\right)= & r\left(\Theta_{r}^{\prime} \cos ^{2} \theta-(\ln R)_{r}^{\prime} \sin \theta \cos \theta\right. \\
& \left.+(\ln R)_{r}^{\prime} \sin \theta \cos \theta+\Theta_{r}^{\prime} \sin ^{2} \theta\right) \\
= & r \Theta_{r}^{\prime},
\end{aligned}
$$

which proves 4.2 .

Notation: Assume $\mathbf{f}: B \subseteq \mathbb{B} \rightarrow \mathbb{B}$ is given. The argument of this function on $\mathbb{B}$ will be demonstrated as $\mathbf{f}(r, \theta)=(R(r, \theta), \Theta(r, \theta))$.

Definition 9. Let $\mathbf{f}: \mathbb{B} \rightarrow \mathbb{B}$ with value $(R(r, \theta), \Theta(r, \theta))$. Assume that $R$ and $\Theta$ have continuous partial derivatives and the Cauchy-Riemann conditions in lemma hold. Then ${ }^{\pi}$ derivative of $\mathbf{f}$ denoted by $\mathbf{f}^{\pi}$ is defined as;

$$
\mathbf{f}^{\pi}(\mathbf{z})=\left(e^{r(\ln R)_{r}^{\prime}}, r \Theta_{r}^{\prime}\right)
$$

$\mathbf{f}$ is said to be ${ }^{\pi}$ analytic on $B \subseteq \mathbb{B}$ if $\mathbf{f}$ is ${ }^{\pi}$ differentiable at any $\mathbf{z} \in B$.

Example 1. Let us prove that the ${ }^{\pi}$ derivative of a ${ }^{\pi}$ constant function $\mathbf{f}(\mathbf{z})=\mathbf{z}_{0}$ is equal to the neutral element $\mathbf{1}=(1,0)$ of multiplication. Here

$$
\mathbf{z}_{0}=\left(r_{0}, \theta_{0}\right)
$$

so

$$
R(r, \theta)=r_{0}
$$

and

$$
\Theta(r, \theta)=\theta_{0} .
$$

Then we have

$$
\mathbf{f}^{\pi}(\mathbf{z})=\left(e^{r(\ln R)_{r}^{\prime}}, r \Theta_{r}^{\prime}\right),
$$

where

$$
(\ln R)_{r}^{\prime}=\Theta_{r}^{\prime}=0 .
$$


Therefore,

$$
\mathbf{f}^{\pi}(z)=\left(e^{0}, 0\right)=(1,0)=\mathbf{1}
$$

Example 2. Consider $\mathbf{a}=\left(r_{1}, \theta_{1}\right)$ and $\mathbf{b}=\left(r_{2}, \theta_{2}\right)$ two constants in $\mathbb{B}$. We know that if $a, b \in \mathbb{C}$, then $\mathbf{a}=\mathbf{E}^{a}$ and $\mathbf{b}=\mathbf{E}^{b}$. Let

$$
f(z)=a z+b,
$$

then the ${ }^{\pi}$ analog of $f(z)$ is;

$$
\begin{aligned}
\mathbf{f}(\mathbf{z}) & =\mathbf{E}^{f(\log \mathbf{z})} \\
& =\mathbf{E}^{a \mathbf{L o g} \mathbf{z}+b} \\
& =\mathbf{E}^{a \mathbf{L o g} \mathbf{z}} \mathbf{E}^{b} \\
& =\mathbf{b} \mathbf{E}^{\log \mathbf{L} \mathbf{L o g} \mathbf{z}} .
\end{aligned}
$$

Now, Let us find ${ }^{\pi}$ derivative of $\mathbf{f}(\mathbf{z})$. In this case we have

$$
\begin{aligned}
\mathbf{f}(\mathbf{z}) & =\mathbf{b} \mathbf{E}^{\mathbf{L o g} \mathbf{a} \mathbf{L o g} \mathbf{z}} \\
& =\left(r_{2}, \theta_{2}\right) \mathbf{E}^{\ln r_{1}+i \theta_{1}} \mathbf{E}^{\ln r+i \theta} \\
& =\left(r_{2}, \theta_{2}\right) \mathbf{E}^{\left(\ln r \ln r_{1}-\theta_{1} \theta\right)+i\left(\theta_{1} \ln r+\theta \ln r_{1}\right)} \\
& =\left(r_{2}, \theta_{2}\right)\left(e^{\ln \ln r_{1}-\theta_{1} \theta}, \theta_{1} \ln r+\theta \ln r_{1}\right) \\
& =\left(r_{2} e^{\ln r_{1} \ln r-\theta_{1} \theta}, \theta \ln r_{1}+\theta_{1} \ln r+\theta_{2}\right) .
\end{aligned}
$$

So,

$$
R(r, \theta)=r_{2} e^{\ln r_{1} \ln r-\theta_{1} \theta}
$$

and

$$
\Theta(r, \theta)=\theta \ln r_{1}+\theta_{1} \ln r+\theta_{2}
$$

Then since,

$$
r(\ln R)_{r}^{\prime}=\ln r_{1}
$$




$$
r \Theta_{r}^{\prime}=\theta_{1},
$$

So,

$$
\begin{aligned}
\mathbf{f}^{\pi}(\mathbf{z}) & =\left(e^{r(\ln R)_{r}^{\prime}}, r \Theta_{r}^{\prime}\right) \\
& =\left(e^{\ln r_{1}}, \theta_{1}\right) \\
& =\left(r_{1}, \theta_{1}\right)
\end{aligned}
$$

$$
=\mathbf{a} \text {. }
$$

Example 3. Consider $\mathbf{f}(\mathbf{z})=\mathbf{E}^{z}$, then

$$
\begin{aligned}
\mathbf{f}(\mathbf{z}) & =\mathbf{E}^{x+i y} \\
& =\left(e^{x}, y\right) \\
& =\left(e^{r \cos \theta}, r \sin \theta\right) .
\end{aligned}
$$

So,

$$
R(r, \theta)=e^{r \cos \theta}
$$

and

$$
\Theta(r, \theta)=r \sin \theta,
$$

and finally since

$$
r(\ln R)_{r}^{\prime}=r \cos \theta
$$

and

$$
r \Theta_{r}^{\prime}=r \sin \theta
$$

we will get

$$
\begin{aligned}
\left(\mathbf{E}^{z}\right)^{\pi} & =\left(e^{r \cos \theta}, r \sin \theta\right)^{\pi} \\
& =\left(e^{r \cos \theta}, r \sin \theta\right) \\
& =\mathbf{E}^{z} .
\end{aligned}
$$

Example 4. Let $\mathbf{f}(\mathbf{z})=\log \mathbf{z}, \mathbf{z} \in \mathbb{B}$, then 


$$
R(r, \theta)=\sqrt{\ln ^{2} r+\theta^{2}}
$$

and

$$
\Theta(r, \theta)=\arctan (\theta, \ln r)
$$

Then since

$$
r(\ln R)_{r}^{\prime}=\frac{\ln r}{\ln ^{2} r+\theta^{2}}
$$

and

$$
r \Theta_{r}^{\prime}=\frac{\theta}{\ln ^{2} r+\theta^{2}} .
$$

Therefore, $(\log \mathbf{z})^{\pi}$ exists and

$$
\begin{aligned}
(\log \mathbf{z})^{\pi} & =\left(e^{\frac{\ln r}{\ln ^{2} r+\theta^{2}}},-\frac{\theta}{\ln ^{2} r+\theta^{2}}\right) \\
& =\mathbf{E}^{\frac{\overline{\log \mathbf{z}}}{\log \mathbf{z} \mid}} \\
& =\mathbf{E}^{\frac{1}{\log \mathbf{z}}} \\
& =\mathbf{1} \oslash \mathbf{z} .
\end{aligned}
$$

which implies

$$
(\log \mathbf{z})^{\pi}=\mathbf{1} \oslash \mathbf{z}
$$

Example 5. In this example we want to verify that,

$$
\begin{aligned}
(\cos z)^{\pi} & =0 \ominus \sin \mathbf{z}, \\
(\sin z)^{\pi} & =\cos \mathbf{z}, \\
(\sinh z)^{\pi} & =\cosh \mathbf{z} \\
(\cosh z)^{\pi} & =\sinh \mathbf{z} .
\end{aligned}
$$


Proof: For $\cos z$ we have

$$
R(r, \theta)=e^{\cosh \theta \cos \ln r}
$$

and

$$
\Theta(r, \theta)=-\sinh \theta \sin \ln r,
$$

which is implying

$$
r(\ln R)_{r}^{\prime}=\Theta_{\theta}^{\prime}=-\cosh \theta \sin \ln r
$$

and

$$
(\ln R)_{\theta}^{\prime}=-r \Theta_{r}^{\prime}=\sinh \theta \cos \ln r .
$$

Therefore, $(\cos \mathbf{z})^{\pi}$ exists with

$$
(\cos \mathbf{z})^{\pi}=\left(e^{-\cosh \theta \sin \ln \theta},-\sinh \theta \cos \ln r\right)=\mathbf{0} \ominus \sin \mathbf{z}
$$

Now, let us prove for $\sin z$. In this case we have

$$
R(r, \theta)=e^{\cosh \theta \sin \ln r}
$$

and

$$
\Theta(r, \theta)=\sinh \theta \cos \ln r,
$$

and we have also

$$
r(\ln R)_{r}^{\prime}=\cosh \theta \cos \ln r
$$

and

$$
r \Theta_{r}^{\prime}=-\sinh \theta \sin \ln r .
$$

So,

$$
\begin{aligned}
(\sin \mathbf{z})^{\pi} & =\left(e^{r(\ln R)_{r}^{\prime}}, r \Theta_{r}^{\prime}\right) \\
& =\left(e^{\cosh \theta \cos \ln r},-\sinh \theta \sin \ln r\right) \\
& =\cos \mathbf{z}
\end{aligned}
$$


The terms of $R(r, \theta)$ and $\Theta(r, \theta)$ for $\sinh \mathbf{z}$ are in the form below;

$$
R(r, \theta)=e^{\cos \theta \sinh \ln r}
$$

and

$$
\Theta(r, \theta)=\sin \theta \cosh \ln r
$$

Using formula above we will get

$$
r(\ln R)_{r}^{\prime}=\cos \theta \cosh \ln r
$$

and

$$
r \Theta_{r}^{\prime}=\sin \theta \sinh \ln r
$$

so

$$
\begin{aligned}
(\sinh \mathbf{z})^{\pi} & =\left(e^{r(\ln R)_{r}^{\prime}}, r \Theta_{r}^{\prime}\right) \\
& =\left(e^{\cos \theta \cosh \ln r}, \sin \theta \sinh \ln r\right) \\
& =\cosh \mathbf{z} .
\end{aligned}
$$

Finally, as same as previous ones for $\cosh \mathbf{z}$ we have

$$
(\cosh z)^{\pi}=\operatorname{sinhz}
$$

which shows all of $\pi$ derivatives of trigonometric bigeometric functions.

Theorem 8. ( The relation between ${ }^{\pi}$ derivative and Newtonian derivative) Let $f$ be a differentiable function at $z \in \mathbb{C}$, then $\mathbf{f}$ which is $\pi$ analog of $f$, is $\pi$ differentiable at $\mathbf{z}=\mathbf{E}^{z} \in \mathbb{B}$ and

$$
\mathbf{f}^{\pi}(\mathbf{z})=\mathbf{E}^{f^{\prime}(\log \mathbf{z})}
$$

Proof: We know that 


$$
\begin{aligned}
\mathbf{f}(r, \boldsymbol{\theta}) & =\mathbf{E}^{f(\mathbf{L o g} \mathbf{z})} \\
& =\mathbf{E}^{f(\ln r+i \theta)} \\
& =\mathbf{E}^{u(\ln r, \theta)+i v(\ln r, \theta)} \\
& =\left(e^{u(\ln r, \theta)}, v(\ln r, \theta)\right) .
\end{aligned}
$$

So,

$$
R(r, \theta)=e^{u(\ln r, \theta)}
$$

and

$$
\Theta(r, \theta)=v(\ln r, \theta) .
$$

Therefore,by Cauchy-Riemann conditions

$$
r(\ln R)_{r}^{\prime}=u_{x}^{\prime}(\ln r, \theta)=v_{y}^{\prime}(\ln r, \theta)=\Theta_{\theta}^{\prime}
$$

and

$$
r \Theta_{r}^{\prime}=v_{x}^{\prime}(\ln r, \theta)=-u_{y}^{\prime}(\ln r, \theta)=-(\ln R)_{\theta}^{\prime} .
$$

and we will get

$$
\begin{aligned}
\mathbf{f}^{\pi}(\mathbf{z}) & =\left(e^{r(\ln R)_{r}^{\prime}}, r \Theta_{r}^{\prime}\right) \\
& =\left(e^{u_{r}^{\prime}(\ln r, \boldsymbol{\theta})}, v_{r}^{\prime}(\ln r, \theta)\right) .
\end{aligned}
$$

And also

$$
f^{\prime}(\log \mathbf{z})=f^{\prime}(\ln r+i \theta)
$$

and we know that

$$
f(z)=f(x, y)=u(x, y)+i v(x, y)
$$

then

$$
f^{\prime}(z)=u_{x}^{\prime}(x, y)+i v_{x}^{\prime}(x, y)
$$

Conseuently,

$$
f^{\prime}(\log \mathbf{z})=u_{r}^{\prime}(\ln r, \theta)+i v_{r}^{\prime}(\ln r, \theta)
$$


After all we will get the result as follows;

$$
\begin{aligned}
\mathbf{E}^{f^{\prime}(\log \mathbf{z})} & =\mathbf{E}^{u_{r}^{\prime}(\ln r, \theta)+i v_{r}^{\prime}(\ln r, \boldsymbol{\theta})} \\
& =\left(e^{u_{r}^{\prime}(\ln r, \theta)}, v_{r}^{\prime}(\ln r, \boldsymbol{\theta})\right) \\
& =\left(e^{r(\ln R)_{r}^{\prime}}, r \Theta_{r}^{\prime}\right) .
\end{aligned}
$$

Theorem 9. Consider that $\mathbf{f}$ and $\mathbf{g}$ are two ${ }^{\pi}$ differentiable functions on $\mathbb{B}$. Then

(a) $\left(\mathbf{z}_{0} \mathbf{f}\right)^{\pi}(\mathbf{z})=\mathbf{f}^{\pi}(\mathbf{z})$ for constant $\mathbf{z}_{0} \in \mathbb{B}$.

(b) $(\mathbf{f g})^{\pi}(\mathbf{z})=\mathbf{f}^{\pi}(\mathbf{z}) \mathbf{g}^{\pi}(\mathbf{z})$.

(c) $(\mathbf{f} / \mathbf{g})^{\pi}(\mathbf{z})=\mathbf{f}^{\pi}(\mathbf{z}) / \mathbf{g}^{\pi}(\mathbf{z})$.

(d) $\mathbf{f}(\mathbf{g}(\mathbf{z}))^{\pi}=\mathbf{f}^{\pi}(\mathbf{g}(\mathbf{z})) \otimes \mathbf{g}^{\pi}(\mathbf{z})$, assuming additionally that $\mathbf{f}^{\pi}(\mathbf{g}(\mathbf{z}))$ exists.

Proof: a) Let $\mathbf{z}_{\mathbf{0}}=\left(r_{0}, \theta_{0}\right)$ be a constant and $\mathbf{f}=(R(r, \theta), \Theta(r, \theta))$, then

$$
\mathbf{z}_{0} \mathbf{f}=\left(r_{0} R(r, \theta), \theta_{0}+\Theta(r, \theta)\right)
$$

which implies that

$$
R_{0}(r, \theta)=r_{0} R(r, \theta)
$$

and

$$
\Theta_{0}(r, \theta)=\theta_{0}+\Theta(r, \theta)
$$

So,

$$
\begin{aligned}
\left(\mathbf{z}_{0} \mathbf{f}\right)^{\pi}(\mathbf{z}) & =\left(e^{r\left(\ln R_{0}\right)_{r}^{\prime}}, r \Theta_{0}^{\prime}\right) \\
& =\left(e^{r\left(\ln r_{0} R(r, \theta)\right)_{r}^{\prime}}, r \Theta_{r}^{\prime}\right) \\
& =\left(e^{r\left(\ln r_{0}+\ln r\right)_{r}^{\prime}}, r \Theta_{r}^{\prime}\right) \\
& =\left(e^{(\ln r)^{\prime} r}, r \Theta_{r}^{\prime}\right) \\
& =\mathbf{f}^{\pi}(\mathbf{z})
\end{aligned}
$$

b) Let $\mathbf{f}=\left(R_{1}, \theta_{1}\right)$ and $\mathbf{g}=\left(R_{2}, \theta_{2}\right)$, then clearly 


$$
\mathbf{f g}=\left(R_{1} R_{2}, \theta_{1}+\theta_{2}\right),
$$

so

$$
R(r, \theta)=R_{1} R_{2}
$$

and

$$
\Theta(r, \theta)=\theta_{1}+\theta_{2}
$$

And then,

$$
\begin{aligned}
(\mathbf{f g})^{\pi}(\mathbf{z}) & =\left(e^{r(\ln R)_{r}^{\prime}}, r \Theta_{r}^{\prime}\right) \\
& =\left(e^{r\left(\ln R_{1}+\ln R_{2}\right)_{r}^{\prime}}, r \Theta_{1 r}^{\prime}+r \Theta_{2 r}^{\prime}\right) \\
& =\left(e^{r\left(\ln R_{1}\right)_{r}^{\prime}} e^{r\left(\ln R_{2}\right)_{r}^{\prime}}, r \Theta_{1 r}^{\prime}+r \Theta_{2 r}^{\prime}\right) \\
& =\left(e^{r\left(\ln R_{1}\right)_{r}^{\prime}}, r \Theta_{1 r}^{\prime}\right)\left(e^{r\left(\ln R_{2}\right)_{r}^{\prime}}, r \Theta_{2 r}^{\prime}\right) \\
& =\mathbf{f}^{\pi}(\mathbf{z}) \mathbf{g}^{\pi}(\mathbf{z}) .
\end{aligned}
$$

c) Let $\mathbf{f}=\left(R_{1}, \theta_{1}\right)$ and $\mathbf{g}=\left(R_{2}, \theta_{2}\right)$, then clearly

$$
\mathbf{f} / \mathbf{g}=\left(R_{1} / R_{2}, \theta_{1}-\theta_{2}\right),
$$

so

$$
R(r, \theta)=R_{1} / R_{2}
$$

and

$$
\Theta(r, \theta)=\theta_{1}-\theta_{2}
$$

And then,

$$
\begin{aligned}
(\mathbf{f} / \mathbf{g})^{\pi}(\mathbf{z}) & =\left(e^{r(\ln R)_{r}^{\prime}}, r \Theta_{r}^{\prime}\right) \\
& =\left(e^{r\left(\ln R_{1}-\ln R_{2}\right)_{r}^{\prime}}, r \Theta_{1 r}^{\prime}-r \Theta_{2 r}^{\prime}\right) \\
& =\left(e^{r\left(\ln R_{1}\right)_{r}^{\prime}} e^{-r\left(\ln R_{2}\right)_{r}^{\prime}}, r \Theta_{1 r}^{\prime}-r \Theta_{2 r}^{\prime}\right) \\
& =\left(e^{r\left(\ln R_{1}\right)_{r}^{\prime}}, r \Theta_{1 r}^{\prime}\right) /\left(e^{r\left(\ln R_{2}\right)_{r}^{\prime}}, r \Theta_{2 r}^{\prime}\right) \\
& =\mathbf{f}^{\pi}(\mathbf{z}) / \mathbf{g}^{\pi}(\mathbf{z}) .
\end{aligned}
$$


d) This part can be proved as follows;

$$
\begin{aligned}
\mathbf{f}(\mathbf{g}((\mathbf{z})))^{\pi} & =\mathbf{E}^{(f \circ g)^{\prime}(\mathbf{L o g} \mathbf{z})} \\
& =\mathbf{E}^{f^{\prime}(g(\mathbf{L o g} \mathbf{z}))} \mathbf{E}^{g^{\prime}(\mathbf{L o g} \mathbf{z})} \\
& =\mathbf{E}^{\mathbf{L o g} \mathbf{E}^{f^{\prime}\left(\log \mathbf{E}^{g(\mathbf{L o g} z)}\right)} \mathbf{g}^{\pi}(\mathbf{z})} \\
& =\mathbf{E}^{\mathbf{L o g} \mathbf{f}^{\pi}(\mathbf{g}(\mathbf{z}))} \mathbf{g}^{\pi}(\mathbf{z}) \\
& =\mathbf{f}^{\pi}(\mathbf{g}(\mathbf{z})) \otimes \mathbf{g}^{\pi}(\mathbf{z}) .
\end{aligned}
$$

\section{$4.4{ }^{\pi}$ Integral}

The motivation of constructing ${ }^{\pi}$ Integral is to have an operator which is inverse of $\pi$ derivative.

Definition 10. Assume $\mathbf{f}: B \subseteq \mathbb{B} \rightarrow \mathbb{B}$ with values

$$
\mathbf{f}(\mathbf{z})=(R(r, \theta), \Theta(r, \theta))
$$

and let $C$ be a contour in $B$ as

$$
C=\mathbf{z}(t)=(r(t), \theta(t)), a \leq t \leq b .
$$

Then the ${ }^{\pi}$ integral of $\mathbf{f}$ along $C$ is defined as;

$$
\int_{C} \mathbf{f}(\mathbf{z})^{\mathbf{d z}}=\mathbf{E}^{\int_{C}\left(\frac{\ln R}{r} d r-\Theta d \theta\right)+i \int_{C}\left(\frac{\Theta}{r} d r+\ln R d \theta\right) .}
$$


Theorem 10. Assume that $\mathbf{f}$ and $\mathbf{g}$ are ${ }^{\pi}$ integrable on a contour $C$. Then

(a) $\int_{C} \mathbf{f}(\mathbf{z})^{\mathbf{d} \mathbf{z}}=\int_{C_{1}} \mathbf{f}(\mathbf{z})^{d \mathbf{z}} \int_{C_{2}} \mathbf{f}(\mathbf{z})^{\mathbf{d z}}$, where $C=C_{1}+C_{2}$.

(b) $\int_{C}(\mathbf{f}(\mathbf{z}) \mathbf{g}(\mathbf{z}))^{\mathbf{d z}}=\int_{C} \mathbf{f}(\mathbf{z})^{\mathbf{d z}} \int_{C} \mathbf{g}(\mathbf{z})^{\mathbf{d z}}$.

(c) $\int_{C}(\mathbf{f}(\mathbf{z}) / \mathbf{g}(\mathbf{z}))^{\mathbf{d z}}=\int_{C} \mathbf{f}(\mathbf{z})^{\mathbf{d z}} / \int_{C} \mathbf{g}(\mathbf{z})^{\mathbf{d z}}$.

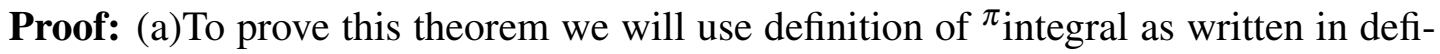
nition above.

$$
\begin{aligned}
\int_{C} \mathbf{f}(\mathbf{z})^{\mathbf{d z}} & =\mathbf{E}^{\int_{C}\left(\frac{\ln R}{r} d r-\Theta d \theta\right)+i \int_{C}\left(\frac{\Theta}{r} d r+\ln R d \theta\right)} \\
& =\mathbf{E}^{\int_{C_{1}+C_{2}}\left(\frac{\ln R}{r} d r-\Theta d \theta\right)+i \int_{C}\left(\frac{\Theta}{r} d r+\ln R d \theta\right)} \\
& =\mathbf{E}^{\left(\int_{C_{1}}\left(\frac{\ln R}{r} d r-\Theta d \theta\right)+i \int_{C_{1}}\left(\frac{\Theta}{r} d r+\ln R d \theta\right)\right)+\left(\int_{C_{2}}\left(\frac{\ln R}{r} d r-\Theta d \theta\right)+i \int_{C_{2}}\left(\frac{\Theta}{r} d r+\ln R d \theta\right)\right)} \\
& =\mathbf{E}^{\left(\int_{C_{1}}\left(\frac{\ln R}{r} d r-\Theta d \theta\right)+i \int_{C_{1}}\left(\frac{\Theta}{r} d r+\ln R d \theta\right)\right)} \mathbf{E}^{\left(\int_{C_{2}}\left(\frac{\ln R}{r} d r-\Theta d \theta\right)+i \int_{C_{2}}\left(\frac{\Theta}{r} d r+\ln R d \theta\right)\right)} \\
& =\int_{C_{1}} \mathbf{f}(\mathbf{z})^{d \mathbf{z}} \int_{C_{2}} \mathbf{f}(\mathbf{z})^{\mathbf{d z}} .
\end{aligned}
$$

(b) Let $\mathbf{f}=\left(R_{1}, \Theta_{1}\right)$ and $\mathbf{g}=\left(R_{2}, \Theta_{2}\right)$ then

$$
\mathbf{f g}=\left(R_{1} R_{2}, \Theta_{1}+\Theta_{2}\right)
$$

So

$$
R(r, \theta)=R_{1} R_{2}
$$

and

$$
\Theta(r, \theta)=\Theta_{1}+\Theta_{2}
$$

and according to the definition of $\pi_{\text {integral, }}$

$$
\begin{aligned}
& \int_{C}(\mathbf{f}(\mathbf{z}) \mathbf{g}(\mathbf{z}))^{\mathbf{d} \mathbf{z}}=\mathbf{E}^{\int_{C}\left(\frac{\ln R_{1} R_{2}}{r} d r-\left(\Theta_{1}+\Theta_{2}\right) d \theta\right)+i \int_{C}\left(\frac{\Theta_{1}+\Theta_{2}}{r} d r+\ln R_{1} R_{2} d \theta\right)}
\end{aligned}
$$

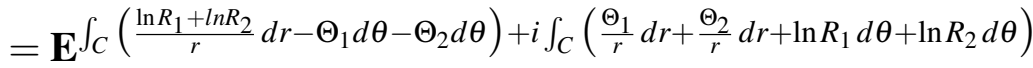

$$
\begin{aligned}
& =\mathbf{E}^{\int_{C}\left(\frac{\ln R_{1}}{r} d r-\Theta_{1} d \theta\right)+i \int_{C}\left(\frac{\Theta_{1}}{r} d r+\ln R_{1} d \theta\right)} \mathbf{E}^{\int_{C}\left(\frac{\ln R_{2}}{r} d r-\Theta_{2} d \theta\right)+i \int_{C}\left(\frac{\Theta_{2}}{r} d r+\ln R_{2} d \theta\right)} \\
& =\int_{C} \mathbf{f}(\mathbf{z})^{\mathbf{d z}} \int_{C} \mathbf{g}(\mathbf{z})^{\mathbf{d z}} .
\end{aligned}
$$


(c) The process is as the same as multiplication as follows;

If $\mathbf{f}=\left(R_{1}, \Theta_{1}\right)$ and $\mathbf{g}=\left(R_{2}, \Theta_{2}\right)$ then

$$
\mathbf{f} \mathbf{g}=\left(R_{1} / R_{2}, \Theta_{1}-\Theta_{2}\right)
$$

so

$$
R(r, \theta)=R_{1} / R_{2}
$$

and

$$
\Theta(r, \theta)=\Theta_{1}-\Theta_{2}
$$

And according to the definition of $\pi_{\text {integral, }}$

$$
\begin{aligned}
\int_{C}(\mathbf{f}(\mathbf{z}) / \mathbf{g}(\mathbf{z}))^{\mathbf{d z}} & =\mathbf{E}^{\int_{C}\left(\frac{\ln R_{1} / R_{2}}{r} d r-\left(\Theta_{1}-\Theta_{2}\right) d \theta\right)+i \int_{C}\left(\frac{\Theta_{1}-\Theta_{2}}{r} d r+\ln R_{1} / R_{2} d \theta\right)} \\
& =\mathbf{E}^{\int_{C}\left(\frac{\ln R_{1}-\ln R_{2}}{r} d r-\Theta_{1} d \theta+\Theta_{2} d \theta\right)+i \int_{C}\left(\frac{\Theta_{1}}{r} d r+\frac{\Theta_{2}}{r} d r+\ln R_{1} d \theta-\ln R_{2} d \theta\right)} \\
& =\mathbf{E}^{\int_{C}\left(\frac{\ln R_{1}}{r} d r-\Theta_{1} d \theta\right)+i \int_{C}\left(\frac{\Theta_{1}}{r} d r+\ln R_{1} d \theta\right)} / \mathbf{E}^{\int_{C}\left(\frac{\ln R_{2}}{r} d r-\Theta_{2} d \theta\right)+i \int_{C}\left(\frac{\Theta_{2}}{r} d r+\ln R_{2} d \theta\right)} \\
& =\int_{C} \mathbf{f}(\mathbf{z})^{\mathbf{d z}} / \int_{C} \mathbf{g}(\mathbf{z})^{\mathbf{d} \mathbf{z}} .
\end{aligned}
$$

As it is mentioned at the beginning of this section, we are looking for the inverse operator of $\pi$ derivative, Now, let us construct $\pi$ analoge of Fundamental Theorem of Calculus.

Theorem 11. (Fundamental Theorem of ${ }^{\pi}$ Calculus) Let $\mathbf{f}: B \subseteq \mathbb{B} \rightarrow \mathbb{B}$ be ${ }^{\pi}$ analytic function and $C$ is a contour in a connected set $B$ with boundary values $\mathbf{z}_{1}$ and $\mathbf{z}_{2}$. Then

$$
\int_{C} \mathbf{f}^{\pi}(\mathbf{z})^{\mathbf{d z}}=\frac{\mathbf{f}\left(\mathbf{z}_{2}\right)}{\mathbf{f}\left(\mathbf{z}_{1}\right)}
$$

Proof: Let

$$
C=\mathbf{z}(t)=(r(t), \theta(t)), a \leq t \leq b,
$$

and

$$
\mathbf{f}(\mathbf{z})=(R(r, \theta), \Theta(r, \theta))
$$


Knowing that

$$
\mathbf{f}^{\pi}(\mathbf{z})=\left(e^{r(\ln R)_{r}^{\prime}}, r \Theta_{r}^{\prime}\right)
$$

so,

$$
R(r, \theta)=e^{r(\ln R)_{r}^{\prime}}
$$

and

$$
\Theta(r, \theta)=r \Theta_{r}^{\prime} .
$$

Using definition of ${ }^{\pi}$ Integral,

$$
\begin{aligned}
\int_{C} \mathbf{f}^{\pi}(\mathbf{z})^{\mathbf{d z}} & =\mathbf{E}^{\int_{C}\left((\ln R)_{r}^{\prime} d r-r \Theta_{r}^{\prime} d \theta\right)+i \int_{C}\left(\Theta_{r}^{\prime} d r+r(\ln R)_{r}^{\prime} d \theta\right)} \\
& =\mathbf{E}^{\int_{C}\left((\ln R)_{r}^{\prime} d r+(\ln R)_{\theta}^{\prime} d \theta\right)+i \int_{C}\left(\Theta_{r}^{\prime} d r+\Theta_{\theta}^{\prime} d \theta\right)} \\
& =\mathbf{E}^{\left.((\ln R)+i \Theta)\right|_{t=a} ^{t=b}}=\left.(R, \Theta)\right|_{t=a} ^{t=b}=\frac{\mathbf{f}\left(\mathbf{z}_{2}\right)}{\mathbf{f}\left(\mathbf{z}_{1}\right)} .
\end{aligned}
$$

Theorem 12. Assume that $\mathbf{f}$ is ${ }^{\pi}$ analytic function on a connected set $B \subseteq \mathbb{B}$ and $C$ is a closed contour on B, then

$$
\oint_{C} \mathbf{f}^{\pi}(\mathbf{z})^{\mathbf{d} \mathbf{z}}=(1,0)=\mathbf{0}
$$

Proof: Again, same as previous theorem

$$
\mathbf{f}^{\pi}(\mathbf{z})=\left(e^{r(\ln R)_{r}^{\prime}}, r \Theta_{r}^{\prime}\right)
$$

which implies that

$$
-\Theta_{r}^{\prime}-\frac{(\ln R)_{\theta}^{\prime}}{r}=(\ln R)_{r}^{\prime}-\frac{\Theta_{\theta}^{\prime}}{r}=0
$$

So,

$$
\begin{aligned}
\int_{C} \mathbf{f}^{\pi}(\mathbf{z})^{\mathbf{d z}} & =\mathbf{E}^{\left.((\ln R)+i \Theta)\right|_{t=a} ^{t=b}} \\
& =\left(e^{0}, 0\right)=(1,0) .
\end{aligned}
$$


Theorem 13. (The relation between ${ }^{\pi}$ integral and Newtonian integral) Assume that $\mathbf{f}: B \subseteq \mathbb{B} \rightarrow \mathbb{B}$ is the ${ }^{\pi}$ analog of the complex function $f$ and $C$ be a contour in $B$. If

$$
\int_{\mathbf{L o g} C} f(z) d z
$$

exists, then

$$
\int_{C} \mathbf{f}(\mathbf{z})^{\mathbf{d z}}
$$

exists such that,

$$
\int_{C} \mathbf{f}(\mathbf{z})^{\mathbf{d z}}=\mathbf{E}^{\int_{\log C} f(z) d z}
$$

Where

$$
\log C=\{\log \mathbf{z}: \mathbf{z} \in C\}
$$

Proof: Define

$$
C=\{\mathbf{z}(t)=(r(t), \theta(t)), a \leq t \leq b\}
$$

Then

$$
\log C=\{z(t)=\ln r(t)+i \theta(t), a \leq t \leq b\}
$$

If

$$
f(x, y)=u(x, y)+i v(x, y)
$$

then its $\pi$ analoge is

$$
\mathbf{f}(\mathbf{z})=(R(r, \theta), \Theta(r, \theta)),
$$

therefore

$$
R(r, \theta)=e^{u(\ln r, \theta)},
$$

And

$$
\Theta(r, \theta)=v(\ln r, \theta) .
$$

Then, 


$$
\begin{aligned}
\int_{\mathbf{L o g} C} f(z) d z= & \int_{\mathbf{L o g} C}(u(x, y)+i v(x, y))(d x+i d y) \\
= & \int_{\mathbf{L o g} C}(u(x, y) d x-v(x, y) d y) \\
& +i \int_{\mathbf{L o g} C}(v(x, y) d x+u(x, y) d y) \\
= & \int_{a}^{b}\left(u(\ln r, \theta) \frac{r^{\prime}}{r}-v(\ln r, \theta) \theta^{\prime}\right) d t \\
& +i \int_{a}^{b}\left(v(\ln r, \theta) \frac{r^{\prime}}{r}+u(\ln r, \theta) \theta^{\prime}\right) d t \\
= & \int_{a}^{b}\left(\frac{\ln R(r, \theta) r^{\prime}}{r}-\Theta(r, \theta) \theta^{\prime}\right) d t \\
& +i \int_{a}^{b}\left(\frac{\Theta(r, \theta) r^{\prime}}{r}+\ln R(r, \theta) \theta^{\prime}\right) d t \\
= & \int_{C}\left(\frac{\ln R(r, \theta)}{r} d r-\Theta(r, \theta) d \theta\right) \\
& +i \int_{C}\left(\frac{\Theta(r, \theta)}{r}+\ln R(r, \theta) d \theta\right) \\
= & \mathbf{E}^{\int_{\mathbf{L o g} C} f(z) d z} .
\end{aligned}
$$

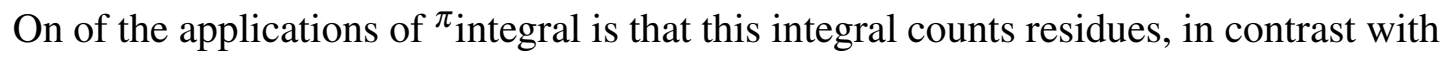
multiplicative integral which was shown in the previous chapter that did not count residue. To show this result, consider example below.

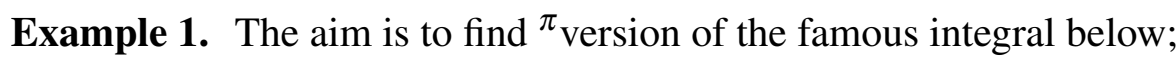

$$
\oint_{|z|=a} \frac{d z}{z}=2 \pi i \quad 0<a .
$$

To do so, consider parametrization of circle as

$$
z(t)=\text { acost }+ \text { iasint }, \quad-\pi \leq t \leq \pi
$$

The ${ }^{\pi}$ version of the written integral is as follows,

$$
\oint_{C}(\mathbf{1} \oslash \mathbf{z})^{\mathbf{d z}}
$$

Clearly, $\mathbf{f}(\mathbf{z})=\mathbf{1} \oslash \mathbf{z}$ has a singularity point at $\mathbf{0}=(1,0)$, so to evaluate if we will follow the last theorem which shows that 


$$
\begin{aligned}
\oint_{C}(\mathbf{1} \oslash \mathbf{z})^{\mathbf{d z}} & =\mathbf{E}^{\oint_{\mathbf{L o g} C} \frac{1}{z} d z} \\
& =\mathbf{E}^{\oint_{|z|=a} \frac{1}{z} d z} \\
& =\mathbf{E}^{2 \pi i}=\left(e^{0}, 2 \pi\right) \\
& =\left(e^{0}, 2 \pi\right) \\
& =(1,2 \pi) .
\end{aligned}
$$

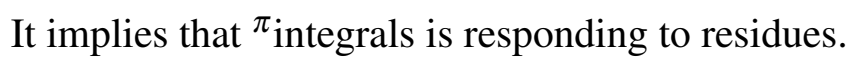




\section{Chapter 5}

\section{CONCLUSION}

Passing through all chapters of this thesis, we tried to show that the Riemann surface of complex logarithm can be transferred to algebraic methods of multiplicative and $\mathrm{Bi}$ geometric calculus without heavy machinery of integral and derivative on Manifolds.

We suggest that, the alternative complex calculus with multiplicative and bi-geometric operations can be more successful and useful for students of engineering and physics who are not going to work with advanced complex analysis. It can be even useful for mathematics students to have different views of complex calculus and Riemann surface of Logarithm.

Altough, we just construct elementary ${ }^{\pi}$ complex calculus, there is no doubts that it can be expanded to the whole by transferring the field $\mathbb{C}$ to the larger field $\mathbb{B}$.

Clearly, the complex ${ }^{\pi}$ calculus is just a part of complex calculus on Riemann surfaces

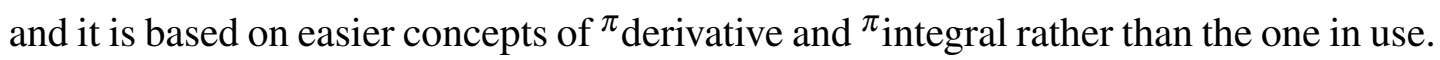
Additionally, the most interesting view of complex ${ }^{\pi}$ calculus is that it always consider the polar representation of elements and functions and all theorems and examples are transferred to polar coordinate which cause many items of complex analysis to be changed and simplified.

We expect that, this new version of complex analysis will be use widely by experts in this field. In this regard, we suggest to investigate and work on Navier-Stokes equations 
that include turbulence property of fluid flow. Since the turbulence appears when the angular velocity of liquid to nonzero, so it is interesting to obtain these equations in term of $r$ and $\theta$ and transfer it to field $\mathbb{B}$. 


\section{REFERENCES}

[1] Grossman, M., \& Katz, R. (1972). Non-Newtonian Calculus: A Self-contained, Elementary Exposition of the Authors' Investigations... Non-Newtonian Calculus.

[2] Bashirov, A. E., Kurpınar, E. M., \& Özyapıcı, A. (2008). Multiplicative calculus and its applications. Journal of Mathematical Analysis and Applications, 337(1), $36-48$

[3] Aniszewska, D. (2007). Multiplicative runge-kutta methods. Nonlinear Dynamics, 50(1), 265-272.

[4] Riza, M., Özyapici, A., \& Misirli, E. (2009). Multiplicative finite difference methods. Quarterly of Applied Mathematics, 67(4), 745-754.

[5] Conway, J. B. (2012). Functions of one complex variable II (Vol. 159). Springer Science \& Business Media.

[6] Brown, J. W. (2009). Complex variables and applications.

[7] Grossman, J., Grossman, M., \& Katz, R. (2006). Averages: a new approach. Non-Newtonian Calculus.

[8] Kadak, U., \& Özlük, M. (2015). Generalized Runge-Kutta method with respect to the non-Newtonian calculus. In Abstract and Applied Analysis (Vol. 2015). Hindawi. 
[9] Boruah, K., \& Hazarika, B. (2017). Application of geometric calculus in numerical analysis and difference sequence spaces. Journal of Mathematical Analysis and Applications, 449(2), 1265-1285.

[10] Misirli, E., \&Gurefe, Y.(2011). Some numerical methods on multiplicative calculus. second International Symposium on Computing in Science \& Engineering, Gediz University, Turkey, Proceeding Number: 700-27.

[11] Abdeljawad, T. (2015). On Multiplicative Fractional Calculus. arXiv preprint arXiv:1510.04176.

[12] Abdeljawad, T., \& Grossman, M. (2016). On geometric fractional calculus. Journal of Semigroup Theory and Applications, 2016, Article-ID.

[13] Bashirov, A. E., \& Riza, M. (2011). On complex multiplicative differentiation. TWMS Journal of Applied and Engineering Mathematics, 1(1), 75.

[14] Bashirov, A. E., \& Norozpour, S. (2013). On complex multiplicative integration. arXiv preprint arXiv:1307.8293.

[15] Uzer, A. (2010). Multiplicative type complex calculus as an alternative to the classical calculus. Computers \& Mathematics with Applications, 60(10), 27252737.

[16] Bashirov, A., \& Riza, M. (2011). Complex multiplicative calculus. arXiv preprint arXiv:1103.1462. 
[17] Bashirov, A. E., \& Norozpour, S. (2016). Riemann surface of complex logarithm and multiplicative calculus. arXiv preprint arXiv:1610.00133.

[18] Filip, D., \& Piatecki, C. (2014). A non-newtonian examination of the theory of exogenous economic growth.

[19] Filip, D., \& Piatecki, C. (2014). An overview on the non-newtonian calculus and its potential applications to economic.

[20] Filip, D., \& Piatecki, C. (2014). In defense of a non-newtonian economic analysis.

[21] Florack, L., \& van Assen, H. (2012). Multiplicative calculus in biomedical image analysis. Journal of Mathematical Imaging and Vision, 42(1), 64-75.

[22] Mora, M., Córdova-Lepe, F., \& Del-Valle, R. (2012). A non-Newtonian gradient for contour detection in images with multiplicative noise. Pattern Recognition Letters, 33(10), 1245-1256.

[23] Abbas, M. U. J. A. H. I. D., Nazir, T., \& Romaguera, S. (2012). Fixed point results for generalized cyclic contraction mappings in partial metric spaces. Revista de la Real Academia de Ciencias Exactas, Fisicas y Naturales. Serie A. Matematicas, 106(2), 287-297.

[24] Türkmen, C., \& Başar, F. (2012, August). Some basic results on the sets of sequences with geometric calculus. In AIP Conference Proceedings (Vol. 1470, No. 1, pp. 95-98). AIP. 
[25] Ozyapici, A., \& Bilgehan, B. (2013, September). Applications of multiplicative calculus to Exponential Signal Processing. In THE ABSTRACT BOOK (p. 165).

[26] Ozyapici, A., \& Bilgehan, B. (2016). Finite product representation via multiplicative calculus and its applications to exponential signal processing. Numerical Algorithms, 71(2), 475-489.

[27] Bilgehan, B. (2015). Efficient approximation for linear and non-linear signal representation. IET Signal Processing, 9(3), 260-266.

[28] Ozyapici, A., \& Bilgehan, B. (2016). Finite product representation via multiplicative calculus and its applications to exponential signal processing. Numerical Algorithms, 71(2), 475-489.

[29] Persch, N., Schroers, C., Setzer, S., \& Weickert, J. (2014, September). Introducing more physics into variational depth-from-defocus. In German Conference on Pattern Recognition (pp. 15-27). Springer, Cham.

[30] Persch, N., Schroers, C., Setzer, S., \& Weickert, J. (2017). Physically inspired depth-from-defocus. Image and Vision Computing, 57, 114-129.

[31] Bashirov, A. E., \& Norozpour, S. On an alternative view to complex calculus. Mathematical Methods in the Applied Sciences. 